ISSN 1855-3966 (printed edn.), ISSN 1855-3974 (electronic edn.)

\author{
ARS MATHEMATICA CONTEMPORANEA 16 (2019) 563-583 \\ https://doi.org/10.26493/1855-3974.793.2a9 \\ (Also available at http://amc-journal.eu)
}

\title{
Classification of some reflexible edge-transitive embeddings of complete bipartite graphs
}

\author{
Jin Ho Kwak \\ Mathematics, Beijing Jiaotong University, Beijing, 100044, P.R. China \\ Young Soo Kwon \\ Mathematics, Yeungnam University, Kyeongsan, 712-749 Republic of Korea
}

Received 8 January 2015, accepted 28 February 2019, published online 21 April 2019

\begin{abstract}
In this paper, we classify some reflexible edge-transitive orientable embeddings of complete bipartite graphs. As a by-product, we classify groups $\Gamma$ such that (i) $\Gamma=X Y$ for some cyclic groups $X=\langle x\rangle$ and $Y=\langle y\rangle$ with $X \cap Y=\left\{1_{\Gamma}\right\}$ and (ii) there exists an automorphism of $\Gamma$ which sends $x$ and $y$ to $x^{-1}$ and $y^{-1}$, respectively.
\end{abstract}

Keywords: Complete bipartite graphs, reflexible edge-transitive embedding.

Math. Subj. Class.: 05C10, 05C30

\section{Preliminaries}

A map is a 2-cell embedding of a graph $G$ in a compact, connected surface. A map is called orientable or nonorientable according to whether the supporting surface is orientable or nonorientable. In this paper, we only consider orientable maps.

For a simple connected graph $G$, an $\operatorname{arc}$ of $G$ is an ordered pair $(u, v)$ of adjacent vertices in $G$. The set of all arcs in $G$ is denoted by $D(G)$. An orientable map $\mathcal{M}$ can be described by a pair $(G ; R)$, where $G$ is the underlying graph of $\mathcal{M}$ and $R$ is a permutation of the arc set $D(G)$ whose orbits coincide with the sets of arcs emanating from the same vertex. The permutation $R$ is called the rotation of the map $\mathcal{M}$.

For given two maps $\mathcal{M}_{1}=\left(G_{1} ; R_{1}\right)$ and $\mathcal{M}_{2}=\left(G_{2} ; R_{2}\right)$, a map isomorphism $\phi: \mathcal{M}_{1} \rightarrow \mathcal{M}_{2}$ is a graph isomorphism $\phi: G_{1} \rightarrow G_{2}$ such that $\phi R_{1}(u, v)=R_{2} \phi(u, v)$ for any $\operatorname{arc}(u, v)$ in $G_{1}$. Furthermore if $\mathcal{M}_{1}=\mathcal{M}_{2}=\mathcal{M}, \phi$ is called a map automorphism of $\mathcal{M}$. The set of all map automorphisms of $\mathcal{M}$ denoted by $\operatorname{Aut}(\mathcal{M})$ is a group under the composition operation, and it is called the automorphism group of $\mathcal{M}$. For a map $\mathcal{M}=(G ; R)$,

E-mail addresses: jinkwak@postech.ac.kr (Jin Ho Kwak), ysookwon@ynu.ac.kr (Young Soo Kwon) 
the group $\operatorname{Aut}(\mathcal{M})$ acts semi-regularly on the arc set $D(G)$, so $|\operatorname{Aut}(\mathcal{M})| \leq 2|E(G)|$. If this bound is attained, then $\operatorname{Aut}(\mathcal{M})$ acts regularly on the arc set, and the map is called a regular map or a regular embedding. The map $\mathcal{M}$ is said to be vertex-transitive or edgetransitive if $\operatorname{Aut}(\mathcal{M})$ acts transitively on $V(G)$ or $E(G)$, respectively. For an orientable embedding $\mathcal{M}$ of a bipartite graph $G$, if the set of partite set preserving map automorphisms acts transitively on $E(G)$ then we call $\mathcal{M}$ an edge-transitive map or an edge-transitive embedding satisfying the Property (P) in this paper. For a map $\mathcal{M}=(G ; R)$, if $\mathcal{M}$ and $\mathcal{M}^{-1}=\left(G ; R^{-1}\right)$ are isomorphic, $\mathcal{M}$ is called reflexible.

Classifying highly symmetric embeddings of graphs in a given class is an interesting problem in topological graph theory. In recent years, there has been particular interest in the regular embeddings of complete bipartite graphs $K_{n, n}$ by several authors [1, 2, 4, 5, 6, 7, $8,10]$. The reflexible regular embeddings and self-Petrie dual regular embeddings of $K_{n, n}$ have been classified by the authors [7]. Recently, G. Jones has completed the classification of regular embeddings of $K_{n, n}$ [5] and the authors have classified nonorientable regular embeddings of $K_{n, n}$ [8]. In [3], Graver and Watkins classified edge-transitive maps on closed surfaces into fourteen types. In this paper, we classify reflexible edge-transitive embeddings of $K_{m, n}$ satisfying the Property (P) which correspond to types 1 or 2 among 14 types. The following theorem is the main result in this paper.

Theorem 1.1. For any integers

$$
\begin{aligned}
m & =2^{a} p_{1}^{a_{1}} \cdots p_{\ell}^{a_{\ell}} p_{\ell+1}^{a_{\ell+1}} \cdots p_{\ell+f}^{a_{\ell+f}} \quad \text { and } \\
n & =2^{b} p_{1}^{b_{1}} \cdots p_{\ell}^{b_{\ell}} q_{\ell+1}^{a_{\ell+1}} \cdots q_{\ell+g}^{b_{\ell+g}} \quad \text { (prime decompositions) }
\end{aligned}
$$

with $\operatorname{gcd}(m, n)=2^{c} p_{1}^{c_{1}} \cdots p_{\ell}^{c_{\ell}}$ and $a \leq b$, the number (up to isomorphism) of reflexible edge-transitive embeddings of $K_{m, n}$ satisfying the Property $(P)$ is 1 if both $m$ and $n$ are odd; $2^{f}\left(1+p_{1}^{c_{1}}\right) \cdots\left(1+p_{\ell}^{c_{\ell}}\right)$ if exactly one of $m$ and $n$ is even, namely, only $n$ is even; $A(a, b) 2^{f+g+\ell}\left(1+p_{1}^{c_{1}}\right) \cdots\left(1+p_{\ell}^{c_{\ell}}\right)$ if both $m$ and $n$ are even, where

$$
A(a, b)= \begin{cases}1 & \text { if }(a, b)=(1,1), \\ 2 & \text { if }(a, b)=(1,2), \\ 4 & \text { if }(a, b)=(2,2) \text { or }(1, k) \text { with } k \geq 3, \\ 10 & \text { if }(a, b)=(2,3), \\ 12 & \text { if }(a, b)=(2, k) \text { with } k \geq 4, \\ 28 & \text { if }(a, b)=(3,3), \\ 40 & \text { if }(a, b)=(3,4), \\ 36 & \text { if }(a, b)=(3, k) \text { with } k \geq 5, \\ 20\left(1+2^{a-2}\right) & \text { if } a=b \geq 4, \\ 20+18 \cdot 2^{a-2} & \text { if } b-1=a \geq 4, \\ 20+16 \cdot 2^{a-2} & \text { if } b-2 \geq a \geq 4 .\end{cases}
$$

Our paper is organized as follows. In the next section, we consider some relations between edge-transitive embeddings of $K_{m, n}$ satisfying the Property $(\mathrm{P})$ and products of two cyclic groups. In Section 3, we classify reflexible edge-transitive embeddings of $K_{m, n}$ satisfying the Property (P) when at least one of $m$ and $n$ is odd. In Section 4, for even integers $m$ and $n$, the classification of reflexible edge-transitive embeddings of $K_{m, n}$ satisfying the Property $(\mathrm{P})$ is given. In the final section, we classify groups $\Gamma$ satisfying the conditions: 
(i) $\Gamma=X Y$ for some cyclic groups $X=\langle x\rangle$ and $Y=\langle y\rangle$ with $X \cap Y=\left\{1_{\Gamma}\right\}$ and

(ii) there exists an automorphism of $\Gamma$ which sends $x$ and $y$ to $x^{-1}$ and $y^{-1}$.

\section{$2(m, n)$-bicyclic triples in $\operatorname{Aut}\left(K_{m, n}\right)$}

Regular embeddings of the complete bipartite graphs $K_{n, n}$ are related to groups $\Gamma$ with two generators satisfying some conditions [4]. Using this relation, G. Jones classify regular embeddings of $K_{n, n}$ [5]. Similarly, we aim to find a relation between edge-transitive embeddings of $K_{m, n}$ satisfying the Property (P) and groups with two generators satisfying some conditions in this section.

In [4], G. Jones et al. showed that any finite group $\Gamma$ is isomorphic to $\operatorname{Aut}(\mathcal{M})$ for some regular embedding $\mathcal{M}$ of $K_{n, n}$ if and only if $\Gamma$ has cyclic subgroups $X=\langle x\rangle$ and $Y=\langle y\rangle$ of order $n$ such that:

(i) $\Gamma=X Y$

(ii) $X \cap Y=\left\{1_{\Gamma}\right\}$ and

(iii) there is an automorphism $\alpha$ of $\Gamma$ transposing $x$ and $y$.

They call the triple $(\Gamma, x, y)$ satisfying these conditions the $n$-isobicyclic triple. In this relation, $x$ and $y$ correspond to rotations of $\mathcal{M}$ around two fixed adjacent vertices $u$ and $v$, respectively. The automorphism $\alpha$ corresponds to the half-turn reversing the edge $u v$. For two $n$-isobicyclic triples $\left(\Gamma_{1}, x_{1}, y_{1}\right)$ and $\left(\Gamma_{2}, x_{2}, y_{2}\right)$, two corresponding regular embeddings $\mathcal{M}_{1}$ and $\mathcal{M}_{2}$ are isomorphic if and only if there exists a group isomorphism from $\Gamma_{1}$ to $\Gamma_{2}$ given by $x_{1} \mapsto x_{2}$ and $y_{1} \mapsto y_{2}$. Using this, one can show that the regular embedding $\mathcal{M}$ induced by $n$-isobicyclic triple $(\Gamma, x, y)$ is reflexible if and only if there exists an automorphism $\beta$ of $\Gamma$ which sends $x$ and $y$ to $x^{-1}$ and $y^{-1}$, respectively. (For more information, the reader is referred to [4].)

Note that one can define an embedding of $K_{n, n}$ by using the first and second conditions of $n$-isobicyclic triple, and the induced map is edge-transitive map satisfying the Property (P) even though the third condition of $n$-isobicyclic triple is not satisfied. Conversely, any edge transitive embedding of $K_{n, n}$ satisfying the Property (P) is isomorphic to some induced map by such a triple $(\Gamma, x, y)$. One can show that for different positive integers $m$ and $n$, an edge-transitive embedding of $K_{m, n}$ satisfying the Property (P) can also be represented by a similar triple. For a group $\Gamma$ containing cyclic subgroups $X=\langle x\rangle$ of order $n$ and $Y=\langle y\rangle$ of order $m$, the triple $(\Gamma, x, y)$ is called $(m, n)$-bicyclic if it satisfies:

(i) $\Gamma=X Y$ and

(ii) $X \cap Y=\left\{1_{\Gamma}\right\}$.

For any $(m, n)$-bicyclic triple $(\Gamma, x, y)$, one can define an embedding of $K_{m, n}$ by a similar way to define an embedding of $K_{n, n}$ using $n$-isobicyclic triple. We denote this embedding by $\mathcal{M}(\Gamma, x, y)$. One can see that $\mathcal{M}(\Gamma, x, y)$ is an edge-transitive embedding of $K_{m, n}$ satisfying the Property (P). Furthermore the following result holds.

Lemma 2.1 ([9]). Let $m$, n be two positive integers (not necessarily distinct).

(1) Any edge-transitive embedding $\mathcal{M}$ of $K_{m, n}$ satisfying the Property $(P)$ is isomorphic to $\mathcal{M}(\Gamma, x, y)$ for some $(m, n)$-bicyclic triple $(\Gamma, x, y)$. 
(2) For two $(m, n)$-bicyclic triples $\left(\Gamma_{1}, x_{1}, y_{1}\right)$ and $\left(\Gamma_{2}, x_{2}, y_{2}\right)$, two edge-transitive embeddings $\mathcal{M}\left(\Gamma_{1}, x_{1}, y_{1}\right)$ and $\mathcal{M}\left(\Gamma_{2}, x_{2}, y_{2}\right)$ are isomorphic if and only if there exists a group isomorphism from $\Gamma_{1}$ to $\Gamma_{2}$ given by $x_{1} \mapsto x_{2}$ and $y_{1} \mapsto y_{2}$.

For any $(m, n)$-bicyclic triple $(\Gamma, x, y)$, there exists a subgroup $H$ of the automorphism group $\operatorname{Aut}\left(K_{m, n}\right)$ such that:

(i) $H$ is isomorphic to $\Gamma$ and

(ii) $x$ and $y$ in $\Gamma$ correspond to elements in $H$ which cyclically permute vertices in the partite sets of size $n$ and $m$, respectively.

Hence it suffices to deal with such $(m, n)$-bicyclic triples in $\operatorname{Aut}\left(K_{m, n}\right)$ to classify edgetransitive embeddings of $K_{m, n}$ satisfying the Property (P).

For any positive integer $m$, denote the set $\{0,1, \ldots, m-1\}$ by $[m]$. Let

$$
V=\{0,1, \ldots,(m-1)\} \cup\left\{0^{\prime}, 1^{\prime}, \ldots,(n-1)^{\prime}\right\}=[m] \cup[n]^{\prime}
$$

be the vertex set of $K_{m, n}$ as partite sets, and let

$$
D=\left\{\left(i, j^{\prime}\right),\left(j^{\prime}, i\right): 0 \leq i \leq m-1 \text { and } 0 \leq j \leq n-1\right\}
$$

be the arc set, where $\left(i, j^{\prime}\right)$ is the arc emanating from $i$ to $j^{\prime}$ and $\left(j^{\prime}, i\right)$ denotes its inverse. We denote the symmetric group on $[m]$ and $[n]^{\prime}$ by $S$ and $S^{\prime}$, respectively. Let $S_{0}$ and $S_{0}^{\prime}$ be their stabilizers of 0 and $0^{\prime}$, respectively. Note that $\operatorname{Aut}\left(K_{m, n}\right)$ is isomorphic to $S \times S^{\prime}$ when $m \neq n ; S \imath \mathbb{Z}_{2}$ when $m=n$. We identify integers $0,1,2, \ldots$ with their residue classes modulo $m$ or $n$ according to the context.

Let $(\Gamma, x, y)$ be an $(m, n)$-bicyclic triple such that $\Gamma$ is a subgroup of $\operatorname{Aut}\left(K_{m, n}\right)$. Now there exists an automorphism $\phi \in \operatorname{Aut}\left(K_{m, n}\right)$ such that

$$
x^{\phi}=\phi^{-1} x \phi=\alpha\left(0^{\prime} 1^{\prime} \cdots(n-1)^{\prime}\right) \quad \text { and } \quad y^{\phi}=\phi^{-1} y \phi=\beta\left(\begin{array}{lll}
0 & 1 & \cdots
\end{array}-1\right),
$$

where $\alpha \in S_{0}$ and $\beta \in S_{0}^{\prime}$. For any $\alpha \in S_{0}$ and $\beta \in S_{0}^{\prime}$, let

$$
x_{\alpha}=\alpha\left(0^{\prime} 1^{\prime} \cdots(n-1)^{\prime}\right) \text { and } y_{\beta}=\beta\left(\begin{array}{llll}
0 & 1 \cdots m-1)
\end{array}\right.
$$

From now on, we only consider triples $\left(\left\langle x_{\alpha}, y_{\beta}\right\rangle, x_{\alpha}, y_{\beta}\right)$ as candidates of $(m, n)$-bicyclic triples.

Lemma 2.2 ([9]). For any $\alpha \in S_{0}$ and $\beta \in S_{0}^{\prime}$,

1. the group $\left\langle x_{\alpha}, y_{\beta}\right\rangle$ acts transitively on the edge set of $K_{m, n}$ and

2. the triple $\left(\left\langle x_{\alpha}, y_{\beta}\right\rangle, x_{\alpha}, y_{\beta}\right)$ is $(m, n)$-bicyclic if and only if $\left|\left\langle x_{\alpha}, y_{\beta}\right\rangle\right|=m n$.

By Lemma 2.2, we need to characterize $\alpha \in S_{0}$ and $\beta \in S_{0}^{\prime}$ satisfying $\left|\left\langle x_{\alpha}, y_{\beta}\right\rangle\right|=$ $m n$ to classify edge-transitive embeddings of $K_{m, n}$ satisfying the Property (P). To do this, we denote

$$
\mathrm{ET}_{m, n}=\left\{(\alpha, \beta): \alpha \in S_{0}, \beta \in S_{0}^{\prime} \text { and }\left|\left\langle x_{\alpha}, y_{\beta}\right\rangle\right|=m n\right\}
$$

Note that for any $(\alpha, \beta) \in \mathrm{ET}_{m, n},\left(\left\langle x_{\alpha}, y_{\beta}\right\rangle, x_{\alpha}, y_{\beta}\right)$ is an $(m, n)$-bicyclic triple and hence $\mathcal{M}\left(\left\langle x_{\alpha}, y_{\beta}\right\rangle, x_{\alpha}, y_{\beta}\right)$ is an edge-transitive embedding of $K_{m, n}$ satisfying the Property (P). Conversely for any edge-transitive embedding $\mathcal{M}$ of $K_{m, n}$ satisfying the Property $(\mathrm{P})$, there exists $(\alpha, \beta) \in \mathrm{ET}_{m, n}$ such that $\mathcal{M}$ is isomorphic to $\mathcal{M}\left(\left\langle x_{\alpha}, y_{\beta}\right\rangle, x_{\alpha}, y_{\beta}\right)$. 


\section{Remark 2.3.}

(1) For any $(\alpha, \beta) \in \mathrm{ET}_{m, n}$,

$$
\left\langle x_{\alpha}, y_{\beta}\right\rangle=\left\{x_{\alpha}^{i} y_{\beta}^{j} \mid i \in[n], j \in[m]\right\}=\left\{y_{\beta}^{j} x_{\alpha}^{i} \mid i \in[n], j \in[m]\right\} .
$$

Hence in many cases, if $\alpha$ satisfies some properties then $\beta$ also satisfies the same properties and vice versa.

(2) Note that for different positive integers $m$ and $n$ and for an orientable embedding $\mathcal{M}$ of $K_{m, n}$, any automorphism of $\mathcal{M}$ is partite set preserving. Let $m=n$ be odd and let $\mathcal{M}$ be an orientable edge-transitive embedding of $K_{n, n}$. If a subgroup $\Gamma$ of $\operatorname{Aut}(\mathcal{M})$ acts regularly on the edge set then $|\Gamma|=m^{2}$ is odd and hence there exists no partite set reversing element in $\Gamma$. Hence for odd $n$, every edge-transitive embedding of $K_{n, n}$ is an edge-transitive embedding of $K_{n, n}$ satisfying the Property (P). On the other hand, for even $n$, we do not know whether the above statement is true or not.

The next lemma shows that for different $\left(\alpha_{1}, \beta_{1}\right),\left(\alpha_{2}, \beta_{2}\right) \in \mathrm{ET}_{m, n}$, two induced edge-transitive embeddings are non-isomorphic.

Lemma 2.4 ([9]). For any $\left(\alpha_{1}, \beta_{1}\right),\left(\alpha_{2}, \beta_{2}\right) \in \mathrm{ET}_{m, n}$, the induced edge-transitive embeddings $\mathcal{M}\left(\left\langle x_{\alpha_{1}}, y_{\beta_{1}}\right\rangle, x_{\alpha_{1}}, y_{\beta_{1}}\right)$ and $\mathcal{M}\left(\left\langle x_{\alpha_{2}}, y_{\beta_{2}}\right\rangle, x_{\alpha_{2}}, y_{\beta_{2}}\right)$ are isomorphic if and only if $\left(\alpha_{1}, \beta_{1}\right)=\left(\alpha_{2}, \beta_{2}\right)$.

By Lemma 2.4, distinct pairs in $\mathrm{ET}_{m, n}$ give non-isomorphic edge-transitive embeddings of $K_{m, n}$ and the number of edge-transitive embeddings of $K_{m, n}$ satisfying the Property (P) equals to the cardinality $\left|\mathrm{ET}_{m, n}\right|$. But for distinct pairs $\left(\alpha_{1}, \beta_{1}\right),\left(\alpha_{2}, \beta_{2}\right) \in$ $\mathrm{ET}_{m, n}$, two groups $\left\langle x_{\alpha_{1}}, y_{\beta_{1}}\right\rangle$ and $\left\langle x_{\alpha_{2}}, y_{\beta_{2}}\right\rangle$ may possibly be isomorphic. We do not know a necessary and sufficient condition for $\left\langle x_{\alpha_{1}}, y_{\beta_{1}}\right\rangle \simeq\left\langle x_{\alpha_{2}}, y_{\beta_{2}}\right\rangle$. So we propose the following problem.

Problem 2.5. For any positive integers $m$ and $n$ and for any $\left(\alpha_{1}, \beta_{1}\right),\left(\alpha_{2}, \beta_{2}\right) \in \mathrm{ET}_{m, n}$, find a necessary and sufficient condition for $\left\langle x_{\alpha_{1}}, y_{\beta_{1}}\right\rangle \simeq\left\langle x_{\alpha_{2}}, y_{\beta_{2}}\right\rangle$.

From now on, we aim to characterize the set $\mathrm{ET}_{m, n}$. Note that for any $(\alpha, \beta) \in \mathrm{ET}_{m, n}$, the stabilizers $\left\langle x_{\alpha}, y_{\beta}\right\rangle_{0}$ and $\left\langle x_{\alpha}, y_{\beta}\right\rangle_{0^{\prime}}$ are cyclic groups $\left\langle x_{\alpha}\right\rangle$ of order $n$ and $\left\langle y_{\beta}\right\rangle$ of order $m$, respectively.

Lemma 2.6. For any $(\alpha, \beta) \in \mathrm{ET}_{m, n},\langle\alpha\rangle$ and $\langle\beta\rangle$ are cyclic groups of order $\left|\left\{\alpha^{i}(1): i \in[n]\right\}\right|$ and $\left|\left\{\beta^{i}\left(1^{\prime}\right): i \in[m]\right\}\right|$, the lengths of the orbit containing 1 and $1^{\prime}$, respectively. Furthermore they are divisors of $n$ and $m$, respectively.

Proof. Let $d_{1}=\left|\left\{\alpha^{i}(1): i \in[n]\right\}\right|$ and $d_{2}=\left|\left\{\beta^{i}\left(1^{\prime}\right): i \in[m]\right\}\right|$. Now $d_{1}$ and $d_{2}$ are divisors of the orders $\left|\left\langle x_{\alpha}\right\rangle\right|=n$ and $\left|\left\langle y_{\beta}\right\rangle\right|=m$, respectively. Note that

$$
\alpha^{d_{1}}(1)=1 \quad \text { and } \quad y_{\beta}^{-1} x_{\alpha}^{d_{1}} y_{\beta}(0)=0,
$$

which implies that, as a conjugate of $x_{\alpha}^{d_{1}}, y_{\beta}^{-1} x_{\alpha}^{d_{1}} y_{\beta}$ belongs to the vertex stabilizer $\left\langle x_{\alpha}, y_{\beta}\right\rangle_{0}=\left\langle x_{\alpha}\right\rangle$. Since $d_{1}$ is a divisor of $n, y_{\beta}^{-1} x_{\alpha}^{d_{1}} y_{\beta}=x_{\alpha}^{r d_{1}}$ for some $r \in[n]$ such that $\operatorname{gcd}\left(r, \frac{n}{d_{1}}\right)=1$, where $\operatorname{gcd}\left(r, \frac{n}{d_{1}}\right)$ is the greatest common divisor of $r$ and $\frac{n}{d_{1}}$. Now, 
suppose to the contrary that $|\langle\alpha\rangle| \neq d_{1}$. Then there exists $k \in[m]$ such that $\alpha^{d_{1}}(k) \neq k$. Let $q$ be the largest element in $[m]$ such that $\alpha^{d_{1}}(q) \neq q$. On the other hand,

$$
\alpha^{r d_{1}}(q)=x_{\alpha}^{r d_{1}}(q)=y_{\beta}^{-1} x_{\alpha}^{d_{1}} y_{\beta}(q)=y_{\beta}^{-1} x_{\alpha}^{d_{1}}(q+1)=y_{\beta}^{-1}(q+1)=q,
$$

contradictory to $\alpha^{r d_{1}}(q) \neq q$. Therefore $|\langle\alpha\rangle|=d_{1}$. Similarly, one can show that $|\langle\beta\rangle|=d_{2}$.

For any $(\alpha, \beta) \in \mathrm{ET}_{m, n}$, it follows from Lemma 2.6 that the length of each cycle in $\alpha$ ( $\beta$, resp.) is a divisor of the length $d_{1}\left(d_{2}\right.$, resp.) of the cycle containing 1 ( $1^{\prime}$, resp.).

From now on we denote $i^{\prime},[n]^{\prime}$ and $\beta\left(i^{\prime}\right)$ simply by $i,[n]$ and $\beta(i)$ for any $i^{\prime} \in[n]^{\prime}$, respectively. The following lemma is related to a characterization of the set $\operatorname{ET}_{m, n}$.

Lemma 2.7 ([9]). Let $\alpha \in S_{0}$ and $\beta \in S_{0}^{\prime}$. Then $(\alpha, \beta) \in \mathrm{ET}_{m, n}$ if and only if for each $i \in[n]$, there exist $a(i) \in[n]$ and $b(i) \in[m]$ such that $\alpha^{i}(k)=\alpha^{a(i)}(k+b(i))-1$ for all $k \in[m]$ and $\beta(t+i)=\beta^{b(i)}(t)+a(i)$ for all $t \in[n]$. In this case, we have $a(i)=\beta(i)$ and $b(i)=-\alpha^{-i}(-1)$.

Note that the equations in Lemma 2.7 is equivalent to $y_{\beta} x_{\alpha}^{i}=x_{\alpha}^{a(i)} y_{\beta}^{b(i)}$. The next lemma gives a characterization of $(\alpha, \beta) \in \mathrm{ET}_{m, n}$ whose induced edge-transitive embedding contains a partite set preserving reflection.

Lemma 2.8 ([9]). For any $(\alpha, \beta) \in \mathrm{ET}_{m, n}, \mathcal{M}\left(\left\langle x_{\alpha}, y_{\beta}\right\rangle, x_{\alpha}, y_{\beta}\right)$ contains a partite set preserving reflection if and only if $\alpha^{-1}(-k)=-\alpha(k)$ for any $k \in[m]$ and $\beta^{-1}(-t)=$ $-\beta(t)$ for any $t \in[n]$.

For our convenience, we denote

$$
\begin{aligned}
& \operatorname{RET}_{m, n}=\left\{(\alpha, \beta) \in \operatorname{ET}_{m, n}: \alpha^{-1}(-k)=-\alpha(k) \text { for any } k \in[m]\right. \text { and } \\
& \left.\qquad \beta^{-1}(-t)=-\beta(t) \text { for any } t \in[n]\right\} .
\end{aligned}
$$

We call an edge-transitive embedding of $K_{m, n}$ satisfying the Property (P) which also contains a partite set preserving reflection a reflexible edge-transitive embedding of $K_{m, n}$ satisfying the Property $(P)$. By Lemmas 2.4 and 2.8, the number (up to isomorphism) of reflexible edge-transitive embeddings of $K_{m, n}$ satisfying the Property (P) equals to the cardinality $\left|\operatorname{RET}_{m, n}\right|$. Note that if $\alpha \in S$ and $\beta \in S^{\prime}$ are the identity permutations, then $(\alpha, \beta)$ belongs to $\operatorname{RET}_{m, n}$ by Lemma 2.8. So for any two positive integers $m$ and $n$, there exists at least one reflexible edge-transitive embeddings of $K_{m, n}$ satisfying the Property (P).

By Lemma 2.8, for any $(\alpha, \beta) \in \operatorname{RET}_{m, n}$ and for any $j \in[m]$ and $i \in[n]$

$$
\alpha^{-i}(-j)=\alpha^{-i+1}(-\alpha(j))=\alpha^{-i+2}\left(-\alpha^{2}(j)\right)=\cdots=\alpha^{-1}\left(-\alpha^{i-1}(j)\right)=-\alpha^{i}(j)
$$

and similarly $\beta^{-j}(-i)=-\beta^{j}(i)$.

Lemma 2.9. For any $(\alpha, \beta) \in \mathrm{RET}_{m, n}$ and for any $j \in[m]$ and $i \in[n]$,

$$
y_{\beta}^{j} x_{\alpha}^{i}=x_{\alpha}^{\beta^{j}(i)} y_{\beta}^{\alpha^{i}(j)}
$$


Proof. Since $\left\langle x_{\alpha}, y_{\beta}\right\rangle=\left\langle x_{\alpha}\right\rangle\left\langle y_{\beta}\right\rangle$, for any $j \in[m]$ and $i \in[n]$, there exist $a(i, j) \in[n]$ and $b(i, j) \in[m]$ such that $y_{\beta}^{j} x_{\alpha}^{i}=x_{\alpha}^{a(i, j)} y_{\beta}^{b(i, j)}$. By taking their values of $k \in[m]$ and $t \in[n]$, we have

$$
\alpha^{i}(k)+j=\alpha^{a(i, j)}(k+b(i, j)) \quad \text { and } \quad \beta^{j}(t+i)=\beta^{b(i, j)}(t)+a(i, j) .
$$

Inserting $k=-b(i, j)$ and $t=0$ to the equation $\alpha^{i}(k)+j=\alpha^{a(i, j)}(k+b(i, j))$ and $\beta^{j}(t+i)=\beta^{b(i, j)}(t)+a(i, j)$, respectively, we have

$$
b(i, j)=-\alpha^{-i}(-j)=\alpha^{i}(j) \quad \text { and } \quad a(i, j)=\beta^{j}(i) .
$$

Lemma 2.10. Let $(\alpha, \beta) \in \operatorname{RET}_{m, n}$ and let $d_{1}=|\langle\alpha\rangle|$ and $d_{2}=|\langle\beta\rangle|$. It holds that $\alpha(k) \equiv-k\left(\bmod d_{2}\right)$ for any $k \in[m]$ and $\beta(t) \equiv-t\left(\bmod d_{1}\right)$ for any $t \in[n]$.

Proof. By Lemma 2.7, for each $i \in[n]$, there exist $a(i) \in[n]$ and $b(i) \in[m]$ such that $\alpha^{i}(k)=\alpha^{a(i)}(k+b(i))-1$ for all $k \in[m]$ and $\beta(t+i)=\beta^{b(i)}(t)+a(i)$ for all $t \in[n]$. Furthermore $a(i)=\beta(i)$ and $b(i)=-\alpha^{-i}(-1)=\alpha^{i}(1)$. Inserting $k=0$ to the equation $\alpha^{i}(k)=\alpha^{a(i)}(k+b(i))-1$, we have $b(i)=\alpha^{-a(i)}(1)=\alpha^{-\beta(i)}(1)$. Hence $\alpha^{i}(1)=\alpha^{-\beta(i)}(1)$ for any $i \in[n]$. Since the order of $\alpha$ equals to the length of the orbit containing 1 by Lemma $2.6, \beta(i) \equiv-i\left(\bmod d_{1}\right)$. By symmetry between $\alpha$ and $\beta$, it also holds that $\alpha(k) \equiv-k\left(\bmod d_{2}\right)$ for any $k \in[m]$.

By Lemmas 2.7 and 2.10, $b(i)=-\alpha^{-i}(-1)=\alpha^{i}(1) \equiv(-1)^{i}\left(\bmod d_{2}\right)$. Hence for any $(\alpha, \beta) \in \operatorname{RET}_{m, n}$ with $d_{1}=|\langle\alpha\rangle|$ and $d_{2}=|\langle\beta\rangle|$, we have

$$
\beta(t+i)=\beta^{b(i)}(t)+a(i)=\beta^{\alpha^{i}(1)}(t)+\beta(i)=\beta^{(-1)^{i}}(t)+\beta(i)
$$

for all $i, t \in[n]$. By symmetry, it also holds $\alpha(k+j)=\alpha^{(-1)^{j}}(k)+\alpha(j)$ for all $j, k \in[m]$.

Lemma 2.11. Let $(\alpha, \beta) \in \operatorname{RET}_{m, n}$ and let $d_{1}=|\langle\alpha\rangle|$ and $d_{2}=|\langle\beta\rangle|$. Now

(1) if one of $d_{1}$ and $d_{2}$ is 1 , say $d_{1}=1$, then either $d_{2}=1$ or ( $m$ is even and $d_{2}=2$ );

(2) if one of $d_{1}$ and $d_{2}$ is at least 3 , say $d_{1} \geq 3$, then both $m$ and $d_{2}$ are even;

(3) if $m(n$, resp.) is even then $\alpha(\beta$, resp.) is parity preserving. Furthermore there exists $s, t \in[m]$ such that $\alpha(2 k)=2 k t, \alpha(2 k+1)=2 k t+2 s+1$ and $2 t^{2}=2$;

(4) if both $d_{1}$ and $d_{2}$ are at least 3 then they are divisors of $\operatorname{gcd}(m, n)$.

Proof. (1): Let $d_{1}=1$ and $d_{2} \geq 2$. By Lemma 2.10, $\alpha(1) \equiv-1\left(\bmod d_{2}\right)$. Since $\alpha$ is the identity, $1 \equiv-1\left(\bmod d_{2}\right)$. By the assumption $d_{2} \geq 2, d_{2}=2$. By Lemma $2.6, d_{2}$ is a divisor of $m$, and hence $m$ is even.

(2): Let $d_{1} \geq 3$. By lemma $2.10, \beta(k) \equiv-k\left(\bmod d_{1}\right)$, which implies that the order $d_{2}$ of $\beta$ is even. Since $d_{2}$ is a divisor of $m, m$ is also even.

(3): Let $m$ be even. If $d_{1}=1$ then $\alpha$ is the identity and hence $\alpha$ is parity preserving. If $d_{1}=2$ then $\alpha^{-1}=\alpha$ and

$$
\alpha(k)=\alpha(k-1+1)=\alpha(k-1)+\alpha(1)=\alpha(k-2)+2 \alpha(1)=\cdots=k \alpha(1)
$$

for all $k \in[m]$. Since $\alpha^{2}(1)=\alpha(\alpha(1))=(\alpha(1))^{2}=1$ and $m$ is even, $\alpha(1)$ should be odd. Hence $\alpha$ is parity preserving. Assume that $d_{1} \geq 3$. Then, $d_{2}$ is even by (2). Since $\alpha(k) \equiv-k\left(\bmod d_{2}\right), \alpha$ is parity preserving. 
For any $2 k \in[m]$,

$$
\begin{aligned}
\alpha(2 k) & =\alpha(2(k-1))+\alpha(2)=\alpha(2(k-2))+2 \alpha(2)=\cdots=k \alpha(2) \quad \text { and } \\
\alpha(2 k+1) & =\alpha(2(k-1)+1)+\alpha(2)=\cdots=\alpha(1)+k \alpha(2) .
\end{aligned}
$$

Let $\alpha(1)=2 s+1$ and $\alpha(2)=2 t$. Now $\alpha(2 k)=k \alpha(2)=2 k t$ and $\alpha(2 k+1)=$ $k \alpha(2)+\alpha(1)=2 k t+2 s+1$. Note that for any $2 k \in[m], \alpha(1)+\alpha(2 k)=\alpha(2 k+$ $1)=\alpha^{-1}(2 k)+\alpha(1)$. Hence $\alpha^{-1}(2 k)=\alpha(2 k)$, namely, $\alpha^{2}(2 k)=2 k$. So we have $\alpha^{2}(2)=\alpha(2 t)=2 t^{2}=2$.

(4): Let $d_{1}, d_{2} \geq 3$. Now all of $d_{1}, d_{2}, m$ and $n$ are even by (2). Hence there exist $s, t \in[m]$ such that $\alpha(2 k)=2 k t, \alpha(2 k+1)=2 k t+2 s+1$ and $2 t^{2}=2$ by (3). Since $d_{1}$ is even and

$$
\alpha^{2 i}(1)=\alpha^{2 i-1}(2 s+1)=\alpha^{2 i-2}(2 s t+2 s+1)=\cdots=2 i s(t+1)+1,
$$

$d_{1}$ is the smallest positive integer such that $d_{1} s(t+1) \equiv 0(\bmod m)$ by Lemma 2.6. Hence $d_{1}$ is a divisor of $m$ and consequently a divisor of $\operatorname{gcd}(m, n)$. Similarly $d_{2}$ is a divisor of $\operatorname{gcd}(m, n)$.

\section{At least one of $m$ and $n$ is odd}

In this section, we classify reflexible edge-transitive embeddings of $K_{m, n}$ satisfying the Property (P) when at least one of $m$ and $n$ is odd. Note that when at least one of $m$ and $n$ is odd, any orientable edge-transitive embedding of $K_{m, n}$ is an edge-transitive embedding satisfying the Property (P). In [9], the second author counted $\left|\mathrm{RET}_{m, n}\right|$ when both $m$ and $n$ are odd as follows.

Theorem 3.1 ([9]). If both $m$ and $n$ are odd then $\left|\mathrm{RET}_{m, n}\right|=1$, namely, there exists only one reflexible edge-transitive embedding of $K_{m, n}$ satisfying the Property $(P)$ up to isomorphism.

In the next theorem, we count $\left|\operatorname{RET}_{m, n}\right|$ when exactly one of $m$ and $n$ is odd. By symmetry, we assume that $m$ is odd.

Theorem 3.2. Let

$$
m=p_{1}^{a_{1}} \cdots p_{\ell}^{a_{\ell}} p_{\ell+1}^{a_{\ell+1}} \cdots p_{\ell+f}^{a_{\ell+f}} \quad \text { (prime factorization) }
$$

be odd and

$$
n=2^{b} p_{1}^{b_{1}} \cdots p_{\ell}^{b_{\ell}} q_{\ell+1}^{b_{\ell+1}} \cdots q_{\ell+g}^{b_{\ell+g}} \quad \text { (prime factorization) }
$$

be even. Let $\operatorname{gcd}(m, n)=p_{1}^{c_{1}} \cdots p_{\ell}^{c_{\ell}}$ with $c_{i} \geq 1$ for any $i=1, \ldots, \ell$. Now

$$
\left|\operatorname{RET}_{m, n}\right|=2^{f}\left(1+p_{1}^{c_{1}}\right) \cdots\left(1+p_{\ell}^{c_{\ell}}\right),
$$

namely, there exist $2^{f}\left(1+p_{1}^{c_{1}}\right) \cdots\left(1+p_{\ell}^{c_{\ell}}\right)$ reflexible edge-transitive embeddings of $K_{m, n}$ satisfying the Property $(P)$ up to isomorphism.

Proof. Let $(\alpha, \beta) \in \operatorname{RET}_{m, n}$ and let $d_{1}=|\langle\alpha\rangle|$ and $d_{2}=|\langle\beta\rangle|$. Suppose that $d_{1} \geq 3$. Then both $d_{2}$ and $m$ are even by Lemma 2.11(2), which is a contradiction. Hence $d_{1}=1$ or 2 . Furthermore for any $k \in[m]$,

$$
\alpha(k)=\alpha^{-1}(k-1)+\alpha(1)=\alpha(k-1)+\alpha(1)=\cdots=k \alpha(1) .
$$


Let $\alpha(1)=r$. Now $\alpha(k)=r k$ and $\alpha^{2}(1)=\alpha(r)=r^{2} \equiv 1(\bmod m)$.

Since $n$ is even, $\beta$ is parity preserving and there exists $s, t \in[n]$ such that $\beta(2 k)=2 k t$, $\beta(2 k+1)=2 k t+2 s+1$ and $2 t^{2}=2$ for any $2 k \in[n]$ by Lemma 2.11(3). If $2 t \neq 2$ then the length of the orbit containing 2 is 2 and hence $d_{2}$ is even. But it can not happen because $m$ is odd. Hence for any $2 k \in[n], \beta(2 k)=2 k, \beta(2 k+1)=2 k+2 s+1$ and for any $i \in[m]$,

$$
\beta^{i}(1)=\beta^{i-1}(2 s+1)=\beta^{i-2}(2 s+2 s+1)=\cdots=2 i s+1 .
$$

Therefore $d_{2}$ is the smallest positive integer such that $2 d_{2} s \equiv 0(\bmod n)$, which implies that $d_{2}$ is a divisor of $n$, and hence $d_{2}$ is a divisor of $\operatorname{gcd}(m, n)=p_{1}^{c_{1}} \cdots p_{\ell}^{c_{\ell}}$.

If $r \equiv 1\left(\bmod p_{i}^{a_{i}}\right)$ for some $i=1,2, \ldots, \ell$, then the fact $\alpha(1)=r \equiv-1\left(\bmod d_{2}\right)$ implies that $p_{i}$ can not be a divisor of $d_{2}$. Hence $p_{i}^{b_{i}}$ should divide $s$, namely, $s \equiv 0$ $\left(\bmod p_{i}^{b_{i}}\right)$. If $r \equiv-1\left(\bmod p_{j}^{a_{j}}\right)$ for some $j=1,2, \ldots, \ell$, then $s \equiv x \cdot p_{j}^{b_{j}-c_{j}}\left(\bmod p_{j}^{b_{j}}\right)$ for some $x$ with $0 \leq x \leq p_{j}^{c_{j}}-1$ because $d_{2}$ is a divisor of $\operatorname{gcd}(m, n)$. Therefore, for any $j=1, \ldots, \ell$, the pair $\left(r\left(\bmod p_{j}^{a_{j}}\right), s\left(\bmod p_{j}^{b_{j}}\right)\right)$ is $(1,0)$ or $\left(-1, x \cdot p_{j}^{b_{j}-c_{j}}\right)$ for some $x$ with $0 \leq x \leq p_{j}^{c_{j}}-1$.

Because $d_{2} \mid \operatorname{gcd}(m, n)$, we have $2 s \equiv 0\left(\bmod 2^{b}\right)$ and for any $k=1,2, \ldots, g$, $s \equiv 0\left(\bmod q_{\ell+k}^{b_{\ell+k}}\right)$. Since $r^{2} \equiv 1(\bmod m), r \equiv \pm 1\left(\bmod p_{\ell+j}^{a_{\ell+j}}\right)$ for any $j=$ $1,2, \ldots f$.

Conversely for any $r \in[m]$ and $s \in[n]$ satisfying the conditions

(i) for any $j=1, \ldots, \ell$, the pair $\left.\left(r\left(\bmod p_{j}^{a_{j}}\right)\right), s\left(\bmod p_{j}^{b_{j}}\right)\right)$ is $(1,0)$ or $\left(-1, x \cdot p_{j}^{b_{j}-c_{j}}\right)$ for some integer $x$ with $0 \leq x \leq p_{j}^{c_{j}}-1$,

(ii) $2 s \equiv 0\left(\bmod 2^{b} q_{\ell+1}^{b_{\ell+1}} \cdots q_{\ell+g}^{b_{\ell+g}}\right)$ and

(iii) for any $j=1,2, \ldots f, r \equiv \pm 1\left(\bmod p_{\ell+j}^{a_{\ell+j}}\right)$,

define $\alpha(k)=r k$ for any $k \in[m]$ and $\beta(2 t)=2 t, \beta(2 t+1)=2 t+2 s+1$ for any $2 t \in[n]$. Note that $\alpha \in S_{0}$ and $\beta \in S_{0}^{\prime}$. Let $d_{1}^{\prime}=|\langle\alpha\rangle|$ and $d_{2}^{\prime}=|\langle\beta\rangle|$. Now $d_{1}^{\prime}=1$ or 2 depending on the value of $r$ and $d_{2}^{\prime}$ is the smallest positive integer satisfying $2 d_{2}^{\prime} s \equiv 0$ $(\bmod n)$. Note that $d_{2}^{\prime}$ divides $\operatorname{gcd}(m, n)$ and $r \equiv-1\left(\bmod d_{2}^{\prime}\right)$. For any $i \in[n]$, let $a(i)=\beta(i)$ and $b(i)=\alpha^{i}(1)=r^{i}$. For the first case, let $i$ be even. Now $a(i)=\beta(i)=i$ and $b(i)=\alpha^{i}(1)=1$. For any $2 t \in[n]$,

$$
\begin{aligned}
\beta(2 t+i) & =2 t+i \quad \text { and } \\
\beta^{b(i)}(2 t)+a(i)=\beta(2 t)+\beta(i) & =2 t+i
\end{aligned}
$$

and

$$
\begin{aligned}
\beta(2 t+1+i) & =2 t+i+2 s+1 \quad \text { and } \\
\beta^{b(i)}(2 t+1)+a(i)=\beta(2 t+1)+\beta(i) & =2 t+2 s+1+i .
\end{aligned}
$$

Hence $\beta(t+i)=\beta^{b(i)}(t)+a(i)$ for any $t \in[n]$. For any $k \in[m]$,

$$
\begin{aligned}
\alpha^{i}(k) & =k \quad \text { and } \\
\alpha^{a(i)}(k+b(i))-1 & =k .
\end{aligned}
$$


Hence $\alpha^{i}(k)=\alpha^{a(i)}(k+b(x))-1$ for any $k \in[m]$.

For the remaining case, let $i$ be odd. Now $a(i)=\beta(i)=i+2 s$ and $b(i)=\alpha^{i}(1)=$ $r \equiv-1\left(\bmod d_{2}^{\prime}\right)$. For any $2 t \in[n]$,

$$
\begin{aligned}
\beta(2 t+i) & =2 t+i+2 s \quad \text { and } \\
\beta^{b(i)}(2 t)+a(i)=\beta^{-1}(2 t)+\beta(i) & =2 t+i+2 s
\end{aligned}
$$

and

$$
\begin{gathered}
\beta(2 t+1+i)=2 t+i+1 \quad \text { and } \\
\beta^{b(i)}(2 t+1)+a(i)=\beta^{-1}(2 t+1)+\beta(i)
\end{gathered}
$$

Hence $\beta(t+i)=\beta^{b(i)}(t)+a(i)$ for any $t \in[n]$. For any $k \in[m]$,

$$
\begin{aligned}
\alpha^{i}(k) & =r k \quad \text { and } \\
\alpha^{a(i)}(k+b(i))-1=\alpha(k+r)-1 & =r k+r^{2}-1=r k .
\end{aligned}
$$

Hence $\alpha^{i}(k)=\alpha^{a(i)}(k+b(i))-1$ for any $k \in[m]$. By Lemma 2.7, $(\alpha, \beta) \in \mathrm{ET}_{m, n}$. Furthermore one can easily check that $\alpha^{-1}(-k)=-\alpha(k)$ for any $k \in[m]$ and $\beta^{-1}(-t)=-\beta(t)$ for any $t \in[n]$. Hence $(\alpha, \beta) \in \operatorname{RET}_{m, n}$ by Lemma 2.8 .

Therefore

$$
\left|\operatorname{RET}_{m, n}\right|=2^{f}\left(1+p_{1}^{c_{1}}\right) \cdots\left(1+p_{\ell}^{c_{\ell}}\right) .
$$

\section{Both $m$ and $n$ are even}

In this section, we classify reflexible edge-transitive embeddings of $K_{m, n}$ satisfying the Property (P) when both $m$ and $n$ are even, and consequently prove Theorem 1.1. For the classification, we give the following lemma.

Lemma 4.1. Let $m$ and $n$ be even and let $\alpha \in S_{0}$ and $\beta \in S_{0}^{\prime}$ with $d_{1}=|\langle\alpha\rangle|$ and $d_{2}=|\langle\beta\rangle|$. Now $(\alpha, \beta) \in \mathrm{RET}_{m, n}$ if and only if $\alpha$ and $\beta$ are defined by

$$
\begin{aligned}
\alpha(2 k) & =2 k t_{1} \quad \text { and } \\
\alpha(2 k+1) & =2 k t_{1}+2 s_{1}+1
\end{aligned}
$$

for any $2 k \in[m]$ and

$$
\begin{aligned}
\beta(2 k) & =2 k t_{2} \quad \text { and } \\
\beta(2 k+1) & =2 k t_{2}+2 s_{2}+1
\end{aligned}
$$

for any $2 k \in[n]$ for some quadruple $\left(s_{1}, t_{1} ; s_{2}, t_{2}\right) \in\left[\frac{m}{2}\right] \times\left[\frac{m}{2}\right] \times\left[\frac{n}{2}\right] \times\left[\frac{n}{2}\right]$ satisfying the following conditions;

(i) $d_{1} \mid \operatorname{gcd}(m, n)$ and $d_{2} \mid \operatorname{gcd}(m, n)$;

(ii) $2 t_{1}^{2} \equiv 2(\bmod m)$ and $2 t_{2}^{2} \equiv 2(\bmod n)$;

(iii) $2\left(s_{1}+1\right) \equiv 0\left(\bmod d_{2}\right), 2\left(t_{1}+1\right) \equiv 0\left(\bmod d_{2}\right)$,

$2\left(s_{2}+1\right) \equiv 0\left(\bmod d_{1}\right)$, and $2\left(t_{2}+1\right) \equiv 0\left(\bmod d_{1}\right)$;

(iv) $2\left(s_{1}+1\right)\left(t_{1}-1\right) \equiv 0(\bmod m)$ and $2\left(s_{2}+1\right)\left(t_{2}-1\right) \equiv 0(\bmod n)$. 
Proof. $(\Leftarrow)$ : Assume that $2 t_{1}=2$, namely, $t_{1}=1$. Then $\alpha(2 k)=2 k$ and $\alpha(2 k+1)=$ $2 k+2 s_{1}+1$ for any $2 k \in[m]$. Since for any $i \in[n], \alpha^{i}(2 k+1)=2 k+2 i s_{1}+1, d_{1}$ is the smallest positive integer such that $2 d_{1} s_{1} \equiv 0(\bmod m)$. Now assume that $2 t_{1} \neq 2$. Then $d_{1}$ should be even because $\alpha^{2}(2)=2 t_{1}^{2}=2$. Since for any $2 i \in[n]$ and for any $2 k \in[m]$, $\alpha^{2 i}(2 k+1)=2 k+2 i s_{1}\left(t_{1}+1\right)+1, d_{1}$ is the smallest positive even integer such that $d_{1} s_{1}\left(t_{1}+1\right) \equiv 0(\bmod m)$. Similarly one can show that $d_{2}$ is the smallest positive integer such that $2 d_{2} s_{2} \equiv 0(\bmod n)$ if $t_{2}=1$; and the smallest positive even integer such that $d_{2} s_{2}\left(t_{2}+1\right) \equiv 0(\bmod n)$ if $t_{2} \neq 1$.

For any $i \in[n]$, let $a(i)=\beta(i)$ and $b(i)=\alpha^{i}(1)$. For the first case, let $i$ be even. Then $a(i)=\beta(i)=i t_{2} \equiv-i\left(\bmod d_{1}\right)$ and $b(i)=\alpha^{i}(1)=i s_{1}\left(t_{1}+1\right)+1 \equiv 1\left(\bmod d_{2}\right)$. For any $2 k \in[n]$,

$$
\begin{aligned}
\beta(2 k+i) & =2 k t_{2}+i t_{2} \quad \text { and } \\
\beta^{b(i)}(2 k)+a(i)=\beta(2 k)+\beta(i) & =2 k t_{2}+i t_{2}
\end{aligned}
$$

and

$$
\begin{aligned}
\beta(2 k+1+i) & =2 k t_{2}+i t_{2}+2 s_{2}+1 \quad \text { and } \\
\beta^{b(i)}(2 k+1)+a(i)=\beta(2 k+1)+\beta(i) & =2 k t_{2}+2 s_{2}+1+i t_{2} .
\end{aligned}
$$

Hence $\beta(k+i)=\beta^{b(i)}(k)+a(i)$ for any $k \in[n]$. For any $2 k \in[m]$,

$$
\begin{aligned}
\alpha^{i}(2 k) & =2 k \text { and } \\
\alpha^{a(i)}(2 k+b(i))-1 & =\alpha^{-i}\left(2 k+i s_{1}\left(t_{1}+1\right)+1\right)-1 \\
& =\left(2 k+i s_{1}\left(t_{1}+1\right)-i s_{1}\left(t_{1}+1\right)+1\right)-1=2 k
\end{aligned}
$$

and

$$
\begin{aligned}
\alpha^{i}(2 k+1) & =2 k+i s_{1}\left(t_{1}+1\right)+1, \quad \text { and } \\
\alpha^{a(i)}(2 k+1+b(i))-1 & =\alpha^{-i}\left(2 k+i s_{1}\left(t_{1}+1\right)+2\right)-1 \\
& =\left(2 k+i s_{1}\left(t_{1}+1\right)+2\right)-1=2 k+i s_{1}\left(t_{1}+1\right)+1 .
\end{aligned}
$$

Hence $\alpha^{i}(k)=\alpha^{a(i)}(k+b(i))-1$ for any $k \in[m]$.

For the remaining case, let $i$ be odd. Now $a(i)=\beta(i)=(i-1) t_{2}+2 s_{2}+1 \equiv-i$ $\left(\bmod d_{1}\right)$ and $b(i)=\alpha^{i}(1)=(i-1) s_{1}\left(t_{1}+1\right)+2 s_{1}+1 \equiv-1\left(\bmod d_{2}\right)$. For any $2 k \in[n]$,

$$
\begin{aligned}
\beta(2 k+i) & =2 k t_{2}+(i-1) t_{2}+2 s_{2}+1 \quad \text { and } \\
\beta^{b(i)}(2 k)+a(i)=\beta^{-1}(2 k)+\beta(i) & =2 k t_{2}+(i-1) t_{2}+2 s_{2}+1
\end{aligned}
$$

and

$$
\begin{aligned}
\beta(2 k+1+i) & =(2 k+i+1) t_{2} \quad \text { and } \\
\beta^{b(i)}(2 k+1)+a(i) & =\beta^{-1}(2 k+1)+\beta(i) \\
& =\left(2 k t_{2}-2 s_{2} t_{2}+1\right)+(i-1) t_{2}+2 s_{2}+1 \\
& =(2 k+i+1) t_{2}-2\left(s_{2}+1\right)\left(t_{2}-1\right)=(2 k+i+1) t_{2} .
\end{aligned}
$$


Hence $\beta(k+i)=\beta^{b(i)}(k)+a(i)$ for any $k \in[n]$. For any $2 k \in[m]$,

$$
\begin{aligned}
\alpha^{i}(2 k) & =2 k t_{1} \quad \text { and } \\
\alpha^{a(i)}(2 k+b(i))-1 & =\alpha^{-i}\left(2 k+(i-1) s_{1}\left(t_{1}+1\right)+2 s_{1}+1\right)-1 \\
& =\left(2 k+(i-1) s_{1}\left(t_{1}+1\right)+2 s_{1}\right) t_{1}-(i+1) s_{1}\left(t_{1}+1\right)+2 s_{1} \\
& =2 k t_{1}-2 s_{1}\left(t_{1}+1\right)+2 s_{1} t_{1}+2 s_{1}=2 k t_{1}
\end{aligned}
$$

and

$$
\begin{aligned}
\alpha^{i}(2 k+1) & =2 k t_{1}+(i-1) s_{1}\left(t_{1}+1\right)+2 s_{1}+1 \quad \text { and } \\
\alpha^{a(i)}(2 k+1+b(i))-1 & =\alpha^{-i}\left(2 k+(i-1) s_{1}\left(t_{1}+1\right)+2 s_{1}+2\right)-1 \\
& =\left(2 k+(i-1) s_{1}\left(t_{1}+1\right)+2 s_{1}+2\right) t_{1}-1 \\
& =2 k t_{1}+(i-1) s_{1}\left(t_{1}+1\right)+2 s_{1}+1+2\left(s_{1}+1\right)\left(t_{1}-1\right) \\
& =2 k t_{1}+(i-1) s_{1}\left(t_{1}+1\right)+2 s_{1}+1 .
\end{aligned}
$$

Hence $\alpha^{i}(k)=\alpha^{a(i)}(k+b(i))-1$ for any $k \in[m]$. By Lemma 2.7, $(\alpha, \beta) \in \mathrm{ET}_{m, n}$. Furthermore one can easily check that $\alpha^{-1}(-k)=-\alpha(k)$ for any $k \in[m]$ and $\beta^{-1}(-k)=$ $-\beta(k)$ for any $k \in[n]$. Hence $(\alpha, \beta) \in \mathrm{RET}_{m, n}$ by Lemma 2.8 .

$(\Rightarrow)$ : Since $m$ and $n$ are even, both $\alpha$ and $\beta$ are parity preserving. For any $2 k \in[m]$,

$$
\begin{aligned}
\alpha(2 k) & =\alpha(2(k-1))+\alpha(2) \\
& =\alpha(2(k-2))+2 \alpha(2)=\cdots=k \alpha(2) \text { and } \\
\alpha(2 k+1) & =\alpha(2(k-1)+1)+\alpha(2) \\
& =\alpha(2(k-2)+1)+2 \alpha(2)=\cdots=\alpha(1)+k \alpha(2) .
\end{aligned}
$$

Let $\alpha(1)=2 s_{1}+1$ and $\alpha(2)=2 t_{1}$ for some $s_{1}, t_{1} \in\left[\frac{m}{2}\right]$. Then $\alpha(2 k)=2 k t_{1}$ and $\alpha(2 k+1)=2 k t_{1}+2 s_{1}+1$ for any $2 k \in[m]$. Note that for any $2 k \in[m], \alpha(1)+\alpha(2 k)=$ $\alpha(2 k+1)=\alpha^{-1}(2 k)+\alpha(1)$. Hence $\alpha^{-1}(2 k)=\alpha(2 k)$, namely, $\alpha^{2}(2 k)=2 k$. It implies that $\alpha^{2}(2)=\alpha\left(2 t_{1}\right)=2 t_{1}^{2} \equiv 2(\bmod m)$. Assume that $2 t_{1}=2$, namely, $t_{1}=1$. Then by Lemma 2.6, the order $|\langle\alpha\rangle|$ is the smallest positive integer $d_{1}$ such that

$$
\alpha^{d_{1}}(1)=\alpha^{d_{1}-1}\left(2 s_{1}+1\right)=\alpha^{d_{1}-2}\left(2 s_{1}+2 s_{1}+1\right)=\cdots=2 d_{1} s_{1}+1 \equiv 1 .
$$

Now assume that $2 t_{1} \neq 2$. Then the order $|\langle\alpha\rangle|$ is even and it is the smallest positive even integer $d_{1}$ such that

$$
\begin{aligned}
\alpha^{d_{1}}(1) & =\alpha^{d_{1}-1}\left(2 s_{1}+1\right)=\alpha^{d_{1}-2}\left(2 s_{1} t_{1}+2 s_{1}+1\right)=\alpha^{d_{1}-3}\left(2 s_{1} t_{1}+4 s_{1}+1\right) \\
& =\alpha^{d_{1}-4}\left(4 s_{1} t_{1}+4 s_{1}+1\right)=\cdots=d_{1} s_{1}\left(t_{1}+1\right)+1 \equiv 1 .
\end{aligned}
$$

Hence $d_{1}$ is a divisor of $m$ and consequently a divisor of $\operatorname{gcd}(m, n)$.

By a similar reason, there exist $s_{2}, t_{2} \in\left[\frac{n}{2}\right]$ such that $\beta(2 k)=2 k t_{2}$ and $\beta(2 k+1)=$ $2 k t_{2}+2 s_{2}+1$ for any $2 k \in[n]$. Furthermore $2 t_{2}^{2} \equiv 2(\bmod n)$ and $d_{2}$ is a divisor of $\operatorname{gcd}(m, n)$. By Lemma 2.10, $\alpha(1)=2 s_{1}+1 \equiv-1\left(\bmod d_{2}\right)$, namely, $2\left(s_{1}+1\right) \equiv 0$ $\left(\bmod d_{2}\right)$ and $\alpha(2)=2 t_{1} \equiv-2\left(\bmod d_{2}\right)$, namely, $2\left(t_{1}+1\right) \equiv 0\left(\bmod d_{2}\right)$. Similarly it holds that $2\left(s_{2}+1\right) \equiv 2\left(t_{2}+1\right) \equiv 0\left(\bmod d_{1}\right)$. Note that

$$
2 t_{1}=\alpha(2)=\alpha^{-1}(1)+\alpha(1)=\left(-2 s_{1} t_{1}+1\right)+2 s_{1}+1 .
$$

Hence $2\left(s_{1}+1\right)\left(t_{1}-1\right) \equiv 0(\bmod m)$. By a similar reason, it holds that $2\left(s_{2}+1\right)\left(t_{2}-1\right) \equiv$ $0(\bmod n)$. 
For even $m$ and $n$, let $\mathcal{Q}(m, n)$ be the set of quadruples $\left(s_{1}, t_{1} ; s_{2}, t_{2}\right) \in\left[\frac{n}{2}\right] \times\left[\frac{n}{2}\right] \times$ $\left[\frac{m}{2}\right] \times\left[\frac{m}{2}\right]$ satisfying the conditions in Lemma 4.1. By Lemma 4.1, the classification of reflexible edge-transitive embeddings of $K_{m, n}$ satisfying the Property (P) is equivalent to the classification of $\mathcal{Q}(m, n)$, and the number $\left|\operatorname{RET}_{m, n}\right|$ equals to the cardinality $|\mathcal{Q}(m, n)|$.

In this section, let

$$
\begin{aligned}
m & =2^{a} p_{1}^{a_{1}} \cdots p_{\ell}^{a_{\ell}} p_{\ell+1}^{a_{\ell+1}} \cdots p_{\ell+f}^{a_{\ell+f}} \quad \text { and } \\
n & =2^{b} p_{1}^{b_{1}} \cdots p_{\ell}^{b_{\ell}} q_{\ell+1}^{a_{\ell+1}} \cdots q_{\ell+g}^{b_{\ell+g}} \quad \text { (prime decompositions) }
\end{aligned}
$$

and let $\operatorname{gcd}(m, n)=2^{c} p_{1}^{c_{1}} \cdots p_{\ell}^{c_{\ell}}$ with $c_{i} \geq 1$ for any $i=1, \ldots, \ell$. Without any loss of generality, assume that $a \leq b$, namely, $a=c$. By Chinese Remainder Theorem, it suffices to consider quadruples $\left(s_{1}, t_{1} ; s_{2}, t_{2}\right)$ modulo prime powers dividing $m$ and $n$, respectively. So we have the following lemma.

Lemma 4.2. For a quadruple $\left(s_{1}, t_{1} ; s_{2}, t_{2}\right) \in\left[\frac{n}{2}\right] \times\left[\frac{n}{2}\right] \times\left[\frac{m}{2}\right] \times\left[\frac{m}{2}\right],\left(s_{1}, t_{1} ; s_{2}, t_{2}\right)$ belongs to $\mathcal{Q}(m, n)$ if and only if:

(1) for $i=1, \ldots, \ell,\left(s_{1}\left(\bmod p_{i}^{a_{i}}\right), t_{1}\left(\bmod p_{i}^{a_{i}}\right) ; s_{2}\left(\bmod p_{i}^{b_{i}}\right), t_{2}\left(\bmod p_{i}^{b_{i}}\right)\right)$ is one of $(-1,-1 ;-1,-1),\left(-1,-1 ; y \cdot p_{i}^{b_{i}-c_{i}}, 1\right),\left(x \cdot p_{i}^{a_{i}-c_{i}}, 1 ;-1,-1\right)$ and $(0,1 ; 0,1)$, where $x, y=0,1, \ldots, p_{i}^{c_{i}}-1$;

(2) for any $j=1,2, \ldots, f,\left(s_{1}\left(\bmod p_{\ell+j}^{a_{\ell+j}}\right), t_{1}\left(\bmod p_{\ell+j}^{a_{\ell+j}}\right)\right)$ is $(0,1)$ or $(-1,-1)$;

(3) for any $k=1,2, \ldots, g,\left(s_{2}\left(\bmod q_{\ell+k}^{b_{\ell+k}}\right), t_{2}\left(\bmod q_{\ell+k}^{b_{\ell+k}}\right)\right)$ is $(0,1)$ or $(-1,-1)$;

(4) $\left(s_{1}\left(\bmod 2^{a}\right), t_{1}\left(\bmod 2^{a}\right) ; s_{2}\left(\bmod 2^{b}\right), t_{2}\left(\bmod 2^{b}\right)\right)$ belongs to $\mathcal{Q}\left(2^{a}, 2^{b}\right)$.

Proof. Assume that $\left(s_{1}, t_{1} ; s_{2}, t_{2}\right)$ belongs to $\mathcal{Q}(m, n)$. Then $t_{1}^{2} \equiv 1\left(\bmod \frac{m}{2}\right)$ and $t_{2}^{2} \equiv 1$ $\left(\bmod \frac{n}{2}\right)$.

(1): First let us consider the quadruple modulo $p_{i}^{a_{i}}$ and $p_{i}^{b_{i}}$ for $i=1, \ldots, \ell$. Note that $t_{1} \equiv \pm 1\left(\bmod p_{i}^{a_{i}}\right)$ and $t_{2} \equiv \pm 1\left(\bmod p_{i}^{b_{i}}\right)$.

If $t_{1} \equiv-1\left(\bmod p_{i}^{a_{i}}\right)$, then $s_{1}$ should be -1 modulo $p_{i}^{a_{i}}$ to satisfy

$$
2\left(s_{1}+1\right)\left(t_{1}-1\right) \equiv 0 \quad\left(\bmod p_{i}^{a_{i}}\right) .
$$

By similar reason, if $t_{2} \equiv-1\left(\bmod p_{i}^{b_{i}}\right)$, then $s_{2} \equiv-1\left(\bmod p_{i}^{b_{i}}\right)$.

Let $\left(s_{1}, t_{1}\right) \equiv(-1,-1)\left(\bmod p_{i}^{a_{i}}\right)$. Since $d_{1}$ is the smallest positive even integer satisfying $d_{1} s_{1}\left(t_{1}+1\right) \equiv 0(\bmod m), p_{i}$ does not divide $d_{1}$. If $t_{2} \equiv-1\left(\bmod p_{i}^{b_{i}}\right)$ then $s_{2}$ should be -1 modulo $p_{i}^{b_{i}}$. If $t_{2} \equiv 1\left(\bmod p_{i}^{b_{i}}\right)$, then $s_{2} \equiv y \cdot p_{i}^{b_{i}-c_{i}}\left(\bmod p_{i}^{b_{i}}\right)$ for some $y=0,1, \ldots, p_{i}^{c_{i}}-1$ because $d_{2} \mid \operatorname{gcd}(m, n)$. By a similar reason, one can say that if $\left(s_{2}, t_{2}\right) \equiv(-1,-1)\left(\bmod p_{i}^{b_{i}}\right)$, then $\left(s_{1}, t_{1}\right) \equiv(-1,-1)$ or $\left(x \cdot p_{i}^{a_{i}-c_{i}}, 1\right)\left(\bmod p_{i}^{a_{i}}\right)$ for some $x=0,1, \ldots, p_{i}^{c_{i}}-1$.

Let $\left(s_{1}, t_{1}\right) \equiv(0,1)\left(\bmod p_{i}^{a_{i}}\right)$. By the condition (iii) in Lemma $4.1, p_{i}$ does not divide $d_{2}$. Note that if $t_{2}=1$ then $d_{2}$ is the smallest positive integer satisfying $2 d_{2} s_{2} \equiv 0$ $(\bmod n)$, and if $t_{2} \neq 1$ then $d_{2}$ is the smallest positive even integer such that $d_{2} s_{2}\left(t_{2}+1\right) \equiv 0(\bmod n)$. Hence $s_{2}=0$ or $t_{2}=-1$ modulo $p_{i}^{b_{i}}$, which implies that $\left(s_{2}, t_{2}\right) \equiv(0,1)$ or $(-1,-1)\left(\bmod p_{i}^{b_{i}}\right)$.

Let $t_{1} \equiv 1\left(\bmod p_{i}^{a_{i}}\right)$ and $s_{1} \neq 0\left(\bmod p_{i}^{a_{i}}\right)$. One can see that $p_{i}$ divides $d_{1}$. By the condition (iii) in Lemma 4.1, $t_{2} \equiv-1\left(\bmod p_{i}^{b_{i}}\right)$ and $s_{2} \equiv-1\left(\bmod p_{i}^{b_{i}}\right)$. 


\section{Therefore}

$\left(s_{1} \quad\left(\bmod p_{i}^{a_{i}}\right), t_{1} \quad\left(\bmod p_{i}^{a_{i}}\right) ; s_{2} \quad\left(\bmod p_{i}^{b_{i}}\right), t_{2} \quad\left(\bmod p_{i}^{b_{i}}\right)\right)=$ $(-1,-1 ;-1,-1),\left(-1,-1 ; y \cdot p_{i}^{b_{i}-c_{i}}, 1\right),\left(x \cdot p_{i}^{a_{i}-c_{i}}, 1 ;-1,-1\right)$ or $(0,1 ; 0,1)$,

where $x, y=0,1, \ldots, p_{i}^{c_{i}}-1$.

(2): For any $j=1,2, \ldots, f, t_{1} \equiv \pm 1\left(\bmod p_{\ell+j}^{a_{\ell+j}}\right)$. If $t_{1} \equiv 1\left(\bmod p_{\ell+j}^{a_{\ell+j}}\right)$ then $s_{1} \equiv 0\left(\bmod p_{\ell+j}^{a_{\ell+j}}\right)$ because $p_{\ell+j}$ does not divide $d_{1}$. If $t_{1} \equiv-1\left(\bmod p_{\ell+j}^{a_{\ell+j}}\right)$ then $s_{1} \equiv-1\left(\bmod p_{\ell+j}^{a_{\ell+j}}\right)$ to satisfy $2\left(s_{1}+1\right)\left(t_{1}-1\right) \equiv 0\left(\bmod p_{\ell+j}^{a_{\ell+j}}\right)$.

(3): By the similar reason with $(2)$, for any $k=1,2, \ldots, g,\left(s_{2}\left(\bmod q_{\ell+k}^{b_{\ell+k}}\right)\right.$, $\left.t_{2}\left(\bmod q_{\ell+k}^{b_{\ell+k}}\right)\right)$ is $(0,1)$ or $(-1,-1)$.

(4): If a quadruple $\left(s_{1}, t_{1} ; s_{2}, t_{2}\right) \in\left[\frac{n}{2}\right] \times\left[\frac{n}{2}\right] \times\left[\frac{m}{2}\right] \times\left[\frac{m}{2}\right]$ satisfies all conditions in Lemma 4.1 , then it also satisfies these conditions modulo $2^{a}$ and $2^{b}$. Hence

$$
\left(s_{1}\left(\bmod 2^{a}\right), t_{1} \quad\left(\bmod 2^{a}\right) ; s_{2} \quad\left(\bmod 2^{b}\right), t_{2} \quad\left(\bmod 2^{b}\right)\right) \in \mathcal{Q}\left(2^{a}, 2^{b}\right) .
$$

By Chinese Remainder Theorem, one can show that if (1), (2), (3) and (4) hold, then $\left(s_{1}, t_{1} ; s_{2}, t_{2}\right) \in \mathcal{Q}(m, n)$.

Corollary 4.3. The number of reflexible edge-transitive embeddings of $K_{m, n}$ satisfying the Property $(P)$ up to isomorphism is $2^{f+g+\ell}\left(1+p_{1}^{c_{1}}\right) \cdots\left(1+p_{\ell}^{c_{\ell}}\right)\left|\mathcal{Q}\left(2^{a}, 2^{b}\right)\right|$.

Proof. By Lemma 4.2, the number of reflexible edge-transitive embeddings of $K_{m, n}$ satisfying the Property $(\mathrm{P})$ up to isomorphism is

$$
\begin{aligned}
&\left(2+2 p_{1}^{c_{1}}\right) \cdots\left(2+2 p_{\ell}^{c_{\ell}}\right) 2^{f} 2^{g}\left|\mathcal{Q}\left(2^{a}, 2^{b}\right)\right|= \\
& 2^{f+g+\ell}\left(1+p_{1}^{c_{1}}\right) \cdots\left(1+p_{\ell}^{c_{\ell}}\right)\left|\mathcal{Q}\left(2^{a}, 2^{b}\right)\right| .
\end{aligned}
$$

By Lemma 4.2, it suffices to classify $\mathcal{Q}\left(2^{a}, 2^{b}\right)$ to classify reflexible edge-transitive embeddings of $K_{m, n}$ satisfying the Property (P). Let $\mathcal{P}(2)=\{(0,1)\}$ and for a 2-power $2^{a}(a>1)$, let $\mathcal{P}\left(2^{a}\right)$ be the set of all pairs $(s, t) \in\left[2^{a-1}\right] \times\left[2^{a-1}\right]$ satisfying the conditions:

(i) $2 t^{2} \equiv 2\left(\bmod 2^{a}\right)$ and

(ii) $2(s+1)(t-1) \equiv 0\left(\bmod 2^{a}\right)$.

For any $(s, t) \in \mathcal{P}\left(2^{a}\right) \backslash\{(0,1)\}$, let $d(s, t)$ be the smallest positive even number $d$ such that $d s(t+1) \equiv 0\left(\bmod 2^{a}\right)$ and let $e(s, t)$ be the largest number $2^{j}$ with $2^{j} \leq 2^{a}$ satisfying $2(s+1) \equiv 0\left(\bmod 2^{j}\right)$ and $2(t+1) \equiv 0\left(\bmod 2^{j}\right)$. Let $d(0,1)=1$ and $e(0,1)=2$. Now we have the following lemma.

Lemma 4.4. For 2-powers $2^{a}(a \geq 1)$ and $2^{b}(b \geq 1)$, a quadruple $\left(s_{1}, t_{1} ; s_{2}, t_{2}\right)$ belongs to $\mathcal{Q}\left(2^{a}, 2^{b}\right)$ if and only if $\left(s_{1}, t_{1} ; s_{2}, t_{2}\right)$ satisfies the conditions

(a) $\left(s_{1}, t_{1}\right) \in \mathcal{P}\left(2^{a}\right)$ and $\left(s_{2}, t_{2}\right) \in \mathcal{P}\left(2^{b}\right)$,

(b) $d\left(s_{1}, t_{1}\right) \leq e\left(s_{2}, t_{2}\right)$ and $d\left(s_{2}, t_{2}\right) \leq e\left(s_{1}, t_{1}\right)$. 
Proof. The conditions (i) and (ii) in the definition of $\mathcal{P}\left(2^{a}\right)$ correspond to the conditions (ii) and (iv) in Lemma 4.1.

Suppose that $d\left(s_{1}, t_{1}\right) \leq e\left(s_{2}, t_{2}\right)$ and $d\left(s_{2}, t_{2}\right) \leq e\left(s_{1}, t_{1}\right)$. Since $d\left(s_{1}, t_{1}\right) \leq 2^{a}$ and $e\left(s_{2}, t_{2}\right) \leq 2^{b}, d\left(s_{1}, t_{1}\right)$ divides $\operatorname{gcd}\left(2^{a}, 2^{b}\right)$, the minimum of $2^{a}$ and $2^{b}$. Similarly $d\left(s_{2}, t_{2}\right)$ also divides $\operatorname{gcd}\left(2^{a}, 2^{b}\right)$. Furthermore it holds that

$$
\begin{aligned}
& 2\left(s_{1}+1\right) \equiv 0 \quad\left(\bmod d\left(s_{2}, t_{2}\right)\right), \\
& 2\left(t_{1}+1\right) \equiv 0 \quad\left(\bmod d\left(s_{2}, t_{2}\right)\right), \\
& 2\left(s_{2}+1\right) \equiv 0 \quad\left(\bmod d\left(s_{1}, t_{1}\right)\right) \quad \text { and } \\
& 2\left(t_{2}+1\right) \equiv 0 \quad\left(\bmod d\left(s_{1}, t_{1}\right)\right) \text {. }
\end{aligned}
$$

Therefore the conditions (i) and (iii) in Lemma 4.1 hold, and hence $\left(s_{1}, t_{1} ; s_{2}, t_{2}\right)$ belongs to $\mathcal{Q}\left(2^{a}, 2^{b}\right)$.

Let $\left(s_{1}, t_{1} ; s_{2}, t_{2}\right)$ belong to $\mathcal{Q}\left(2^{a}, 2^{b}\right)$. Now the condition (iii) in Lemma 4.1 is equivalent to the condition $d\left(s_{1}, t_{1}\right) \leq e\left(s_{2}, t_{2}\right)$ and $d\left(s_{2}, t_{2}\right) \leq e\left(s_{1}, t_{1}\right)$.

By Lemma 4.4, the calculation of $d(s, t)$ and $e(s, t)$ for each $(s, t) \in \mathcal{P}\left(2^{a}\right)$ is helpful to calculate $\left|\mathcal{Q}\left(2^{a}, 2^{b}\right)\right|$. The following lemma gives full list of $(s, t) \in \mathcal{P}\left(2^{a}\right)$ and corresponding $d(s, t)$ and $e(s, t)$.

Lemma 4.5. For a 2-power $2^{a}(a>1)$, the set $\left\{(s, t, d(s, t), e(s, t)):(s, t) \in \mathcal{P}\left(2^{a}\right)\right\}$ is the following:

$$
\begin{cases}\{(0,1,1,2),(1,1,2,4)\}, & \text { if } a=2 \\ \{(0,1,1,2),(1,1,4,4),(2,1,2,2),(3,1,4,4),(1,3,2,4),(3,3,2,8)\}, & \text { if } a=3 \\ \left\{(0,1,1,2),\left(2^{a-2}-1,2^{a-2}-1,4,2^{a-1}\right),\left(2^{a-1}-1,2^{a-2}-1,4,2^{a-1}\right),\right. & \\ \left.\left(2^{a-2}-1,2^{a-1}-1,2,2^{a-1}\right),\left(2^{a-1}-1,2^{a-1}-1,2,2^{a}\right)\right\} & \\ \cup\left\{\left(x, 1,2^{a-1}, 4\right),\left(x, 2^{a-2}+1,2^{a-1}, 4\right): x=1,3, \ldots, 2^{a-1}-1\right\} & \\ \cup\left\{\left(2^{i} y, 1,2^{a-i-1}, 2\right): i=1, \ldots, a-2, y=1,3, \ldots, 2^{a-i-1}-1\right\} & \text { if } a \geq 4 .\end{cases}
$$

Proof. Let $(s, t) \in \mathcal{P}\left(2^{a}\right)$.

For $a=2, t$ should be 1 and both $s=0$ and $s=1$ satisfy the conditions for $(s, t) \in$ $\mathcal{P}\left(2^{a}\right)$. Hence $(s, t, d(s, t), e(s, t))=(0,1,1,2)$ or $(1,1,2,4)$. Let $a=3$. Then $t=1$ and $t=3$. If $t=1$, then $s=i$ for some $i=0,1,2,3$. If $t=3$, then $s=1$ or $s=3$. In any possible pair $(s, t)$, one can easily calculate $d(s, t)$ and $e(s, t)$.

Now assume that $a \geq 4$. Then $t=1,2^{a-2}-1,2^{a-2}+1$ or $2^{a-1}-1$. For $t=1$, any number $0,1,2, \ldots, 2^{a-1}-1$ is possible for $s$ to satisfy the condition (ii) in the definition of $\mathcal{P}\left(2^{a}\right)$. Note that if $(s, t)=(0,1)$, then $(d(0,1), e(0,1))=(1,2)$. One can easily show that if $(s, t)=(x, 1)$ for any $x=1,3, \ldots, 2^{a-1}-1$ then $(d(s, t), e(s, t))=\left(2^{a-1}, 4\right)$. If $(s, t)=\left(2^{i} y, 1\right)$ for any $i=1, \ldots, a-2$ and for any $y=1,3, \ldots, 2^{a-i-1}-1$, then $(d(s, t), e(s, t))=\left(2^{a-i-1}, 2\right)$.

For $t=2^{a-2}-1$, both $s=2^{a-2}-1$ and $s=2^{a-1}-1$ satisfy the conditions for $(s, t) \in \mathcal{P}\left(2^{a}\right)$. If $(s, t)=\left(2^{a-2}-1,2^{a-2}-1\right)$ or $\left(2^{a-1}-1,2^{a-2}-1\right)$ then we have $(d(s, t), e(s, t))=\left(4,2^{a-1}\right)$.

Let $t=2^{a-2}+1$. Then any number $s=1,3, \ldots, 2^{a-1}-1$ satisfies the condition (ii) in the definition of $\mathcal{P}\left(2^{a}\right)$. For any $(s, t)=\left(x, 2^{a-2}+1\right)$ with $x=1,3, \ldots, 2^{a-1}-1$, we have $(d(s, t), e(s, t))=\left(2^{a-1}, 4\right)$. 
For the final case, let $t=2^{a-1}-1$. Then $s=2^{a-2}-1$ or $2^{a-1}-1$. If $(s, t)=\left(2^{a-2}-1\right.$, $\left.2^{a-1}-1\right)$ then we have $(d(s, t), e(s, t))=\left(2,2^{a-1}\right)$; if $(s, t)=\left(2^{a-1}-1,2^{a-1}-1\right)$ then $(d(s, t), e(s, t))=\left(2,2^{a}\right)$.

Theorem 4.6. For any 2-powers $2^{a}$ and $2^{b}$ with $a \leq b$, the number $\left|\mathcal{Q}\left(2^{a}, 2^{b}\right)\right|$ of reflexible edge-transitive embeddings of $K_{m, n}$ satisfying the Property $(P)$ up to isomorphism is the following:

$$
\left|\mathcal{Q}\left(2^{a}, 2^{b}\right)\right|= \begin{cases}1 & \text { if }(a, b)=(1,1), \\ 2 & \text { if }(a, b)=(1,2), \\ 4 & \text { if }(a, b)=(2,2) \text { or }(1, k) \text { with } k \geq 3, \\ 10 & \text { if }(a, b)=(2,3), \\ 12 & \text { if }(a, b)=(2, k) \text { with } k \geq 4, \\ 40 & \text { if }(a, b)=(3,3), \\ 36 & \text { if }(a, b)=(3,4), \\ 20\left(1+2^{a-2}\right) & \text { if }(a, b)=(3, k) \text { with } k \geq 5, \\ 20+18 \cdot 2^{a-2} & \text { if } b-1=a \geq 4, \\ 20+16 \cdot 2^{a-2} & \text { if } b-2 \geq a \geq 4 .\end{cases}
$$

Proof. By Lemma 4.4, it suffices to find all $\left(s_{1}, t_{1} ; s_{2}, t_{2}\right)$ satisfying the conditions

(a) $\left(s_{1}, t_{1}\right) \in \mathcal{P}\left(2^{a}\right)$ and $\left(s_{2}, t_{2}\right) \in \mathcal{P}\left(2^{b}\right)$,

(b) $d\left(s_{1}, t_{1}\right) \leq e\left(s_{2}, t_{2}\right)$ and $d\left(s_{2}, t_{2}\right) \leq e\left(s_{1}, t_{1}\right)$.

By Lemma 4.5, one can get all the lists of $\left(s_{1}, t_{1} ; s_{2}, t_{2}\right)$ satisfying the conditions as Table 1.

Proof of Theorem 1.1. For odd $m$ and $n$, the number $\left|\mathrm{RET}_{m, n}\right|$ of reflexible edge-transitive embeddings of $K_{m, n}$ up to isomorphism is 1 by Theorem 3.1. When exactly one of $m$ and $n$ is odd, then the number $\left|\mathrm{RET}_{m, n}\right|$ is counted in Theorem 3.2.

Assume that both $m$ and $n$ are even. Let

$$
\begin{aligned}
& m=2^{a} p_{1}^{a_{1}} p_{2}^{a_{2}} \cdots p_{\ell}^{a_{\ell}} p_{\ell+1}^{a_{\ell+1}} \cdots p_{\ell+f}^{a_{\ell+f}} \quad \text { and } \\
& n=2^{b} p_{1}^{b_{1}} p_{2}^{b_{2}} \cdots p_{\ell}^{b_{\ell}} q_{\ell+1}^{a_{\ell+1}} \cdots q_{\ell+g}^{b_{\ell+g}} \quad \text { (prime decompositions) }
\end{aligned}
$$

and let $\operatorname{gcd}(m, n)=2^{c} p_{1}^{c_{1}} p_{2}^{c_{2}} \cdots p_{\ell}^{c_{\ell}}$ with $c_{i} \geq 1$ for any $i=1, \ldots, \ell$. Without any loss of generality, assume that $a \leq b$, namely, $a=c$. By Corollary 4.3, the number $\left|\mathrm{RET}_{m, n}\right|=|\mathcal{Q}(m, n)|$ is

$$
2^{f+g+\ell}\left(1+p_{1}^{c_{1}}\right) \cdots\left(1+p_{\ell}^{c_{\ell}}\right)\left|\mathcal{Q}\left(2^{a}, 2^{b}\right)\right| .
$$

Theorem 4.6 completes the proof. 
Table 1: All lists of $\mathcal{Q}\left(2^{a}, 2^{b}\right)$.

\begin{tabular}{c|l}
$(a, b)$ & $\mathcal{Q}\left(2^{a}, 2^{b}\right)$ \\
\hline \hline$(1,1)$ & $(0,1 ; 0,1)$ \\
\hline$(1,2)$ & $(0,1 ; 0,1),(0,1 ; 1,1)$ \\
\hline$(1, \geq 3)$ & $(0,1 ; 0,1),\left(0,1 ; 2^{b-2}, 1\right),\left(0,1 ; 2^{b-2}-1,2^{b-1}-1\right)$, \\
& $\left(0,1 ; 2^{b-1}-1,2^{b-1}-1\right)$ \\
\hline$(2,2)$ & $(0,1 ; 0,1),(0,1 ; 1,1),(1,1 ; 0,1),(1,1 ; 1,1)$ \\
\hline$(2,3)$ & $(0,1 ; 0,1),(0,1 ; 2,1),(0,1 ; 1,3),(0,1 ; 3,3),(1,1 ; 0,1),(1,1 ; 1,1)$, \\
& $(1,1 ; 2,1),(1,1 ; 3,1),(1,1 ; 1,3),(1,1 ; 3,3)$ \\
\hline$(2, \geq 4)$ & $(0,1 ; 0,1),\left(0,1 ; 2^{b-2}, 1\right),\left(0,1 ; 2^{b-2}-1,2^{b-1}-1\right)$, \\
& $\left(0,1 ; 2^{b-1}-1,2^{b-1}-1\right),(1,1 ; 0,1),\left(1,1 ; 2^{b-3}, 1\right),\left(1,1 ; 2^{b-2}, 1\right)$, \\
& $\left(1,1 ; 3 \cdot 2^{b-3}, 1\right),\left(1,1 ; 2^{b-2}-1,2^{b-2}-1\right),\left(1,1 ; 2^{b-1}-1,2^{b-2}-1\right)$, \\
& $\left(1,1 ; 2^{b-2}-1,2^{b-1}-1\right),\left(1,1 ; 2^{b-1}-1,2^{b-1}-1\right)$ \\
\hline$(3,3)$ & $(0$ or 2,$1 ; 0,1),(0$ or 2,$1 ; 2,1),(0$ or 2,$1 ; 1,3),(0$ or 2,$1 ; 3,3)$, \\
& $(1$ or 3,$1 ; 1,1),(1$ or 3,$1 ; 3,1),(1$ or 3,$1 ; 1,3),(1$ or 3,$1 ; 3,3)$, \\
& $(1$ or 3,$3 ; 0,1),(1$ or 3,$3 ; 1,1),(1$ or 3,$3 ; 2,1),(1$ or 3,$3 ; 3,1)$, \\
& $(1$ or 3,$3 ; 1,3),(1$ or 3,$3 ; 3,3)$ \\
\hline$(3,4)$ & $(0$ or 2,$1 ; 0,1),(0$ or 2,$1 ; 4,1),(0$ or 2,$1 ; 3,7),(0$ or 2,$1 ; 7,7)$, \\
& $(1$ or 3,$1 ; 3,3),(1$ or 3,$1 ; 7,3),(1$ or 3,$1 ; 3,7),(1$ or 3,$1 ; 7,7) ;$ \\
& $(1,3 ; x, 1), x=0,2,4,6 ;$ \\
& $(0$ or 2,$1 ; 0,1),\left(0\right.$ or 2,$\left.1 ; 2^{b-2}, 1\right) ;$ \\
& $\left(0\right.$ or 2,$\left.1 ; x, 2^{b-1}-1\right), x=2^{b-2}-1$ or $2^{b-1}-1 ;$ \\
& $(1$ or 3,$1 ; x, y), x, y=2^{b-2}-1$ or $2^{b-1}-1 ;$ \\
& $\left(1,3 ; i \cdot 2^{b-3}, 1\right), i=0,1,2,3 ;$ \\
& $(1,3 ; x, y), x, y=2^{b-2}-1$ or $2^{b-1}-1 ;$ \\
& $\left(3,3 ; i \cdot 2^{b-4}, 1\right), i=0,1, \ldots, 7 ;$ \\
& $(3,3 ; x, y), x, y=2^{b-2}-1$ or $2^{b-1}-1$ \\
\hline$(3, \geq 5)$ & $\left(0\right.$ or $\left.2^{a-2}, 1 ; x, y\right)$, \\
& $(x, y)=(0,1),\left(2^{b-2}, 1\right),\left(2^{b-2}-1,2^{b-1}-1\right)$ or $\left(2^{b-1}-1,2^{b-1}-1\right) ;$ \\
& $\left(2 x, 1 ; 2^{b-2}-1,2^{b-1}-1\right),\left(2 x, 1 ; 2^{b-1}-1,2^{b-1}-1\right)$, \\
& $x=1,2, \ldots, 2^{a-2}-1\left(x \neq 2^{a-3}\right) ;$ \\
& $\left(x, 1\right.$ or $\left.2^{a-2}+1 ; y, z\right)$, \\
& $x=1,3, \ldots, 2^{a-1}-1, y, z=2^{b-2}-1$ or $2^{b-1}-1 ;$ \\
& $\left(2^{a-2}-1\right.$ or $2^{a-1}-1,2^{a-2}-1$ or $\left.2^{a-1}-1 ; x, y\right)$, \\
& $x, y=2^{b-2}-1$ or $2^{b-1}-1 ;$ \\
& $\left(2^{a-2}-1,2^{a-1}-1 ; i \cdot 2^{b-a}, 1\right), i=0,1, \ldots, 2^{a-1}-1 ;$ \\
& 0 \\
&
\end{tabular}

Only when $a=b$ :

$\left(2^{a-2}-1\right.$ or $2^{a-1}-1,2^{a-2}-1 ; x, 1$ or $\left.2^{b-2}+1\right), x=1,3, \ldots, 2^{b-1}-1$;

Only when $a=b$ :

$\left(2^{a-2}-1,2^{a-1}-1 ; x, 2^{b-2}+1\right), x=1,3, \ldots, 2^{b-1}-1 ;$

Only when $a=b$ or $b=a+1$ :

$\left(2^{a-1}-1,2^{a-1}-1 ; x, 1\right), x=0,1, \ldots, 2^{b-1}-1 ;$

Only when $a=b$ or $b=a+1$ :

$\left(2^{a-1}-1,2^{a-1}-1 ; x, 2^{b-2}+1\right), x=1,3, \ldots, 2^{b-1}-1$;

Only when $b \geq a+2$ :

$\left(2^{a-1}-1,2^{a-1}-1 ; i \cdot 2^{b-a-1}, 1\right), i=0,1, \ldots, 2^{a}-1$ 


\section{Classification of some groups}

In this section, we aim to consider a presentation of the group $\left\langle x_{\alpha}, y_{\beta}\right\rangle$ for any $(\alpha, \beta) \in$ $\mathrm{RET}_{m, n}$. And we give some sufficient conditions and necessary conditions for $\left\langle x_{\alpha_{1}}, y_{\beta_{1}}\right\rangle$ and $\left\langle x_{\alpha_{2}}, y_{\beta_{2}}\right\rangle$ to be isomorphic for any $\left(\alpha_{1}, \beta_{1}\right),\left(\alpha_{2}, \beta_{2}\right) \in \mathrm{RET}_{m, n}$. For any positive integers $m$ and $n$, a group $\Gamma$ such that

(i) $\Gamma=X Y$ for some cyclic groups $X=\langle x\rangle$ of order $n$ and $Y=\langle y\rangle$ of order $m$ with $X \cap Y=\left\{1_{\Gamma}\right\}$ and

(ii) there exists an automorphism of $\Gamma$ which sends $x$ and $y$ to $x^{-1}$ and $y^{-1}$, respectively, is isomorphic to $\left\langle x_{\alpha}, y_{\beta}\right\rangle$ for some $(\alpha, \beta) \in \mathrm{RET}_{m, n}$. For our convenience, call a group $\Gamma$ satisfying the conditions (i) and (ii) in the above sentence a reflexible product of two cyclic groups of order $m$ and $n$. Now to classify reflexible products of two cyclic groups of order $m$ and $n$, it suffices to consider $\left\langle x_{\alpha}, y_{\beta}\right\rangle$, where $(\alpha, \beta) \in \operatorname{RET}_{m, n}$. Note that for any integers $i, j$ and for any $(\alpha, \beta) \in \operatorname{RET}_{m, n}$,

$$
y_{\beta}^{i} x_{\alpha}^{j}=x_{\alpha}^{\beta^{i}(j)} y_{\beta}^{\alpha^{j}(i)} .
$$

For example, $y_{\beta} x_{\alpha}=x_{\alpha}^{\beta(1)} y_{\beta}^{\alpha(1)}$ and $y_{\beta} x_{\alpha}^{2}=x_{\alpha}^{\beta(2)} y_{\beta}^{\alpha^{2}(1)}$.

For odd integers $m$ and $n$, since $\operatorname{RET}_{m, n}=\{(\mathrm{id}, \mathrm{id})\}$, there is a unique reflexible product of two cyclic groups of order $m$ and $n$ up to isomorphism, namely, an abelian group $\mathbb{Z}_{m} \times \mathbb{Z}_{n}$.

Let

$$
m=p_{1}^{a_{1}} p_{2}^{a_{2}} \cdots p_{\ell}^{a_{\ell}} p_{\ell+1}^{a_{\ell+1}} \cdots p_{\ell+f}^{a_{\ell+f}} \quad \text { (prime factorization) }
$$

be odd and

$$
n=2^{b} p_{1}^{b_{1}} p_{2}^{b_{2}} \cdots p_{\ell}^{b_{\ell}} q_{\ell+1}^{b_{\ell+1}} \cdots q_{\ell+g}^{b_{\ell+g}} \quad \text { (prime factorization) }
$$

be even. Let $\operatorname{gcd}(m, n)=p_{1}^{c_{1}} p_{2}^{c_{2}} \cdots p_{\ell}^{c_{\ell}}$ with $c_{i} \geq 1$ for any $i=1, \ldots, \ell$. Now $\left|\operatorname{RET}_{m, n}\right|=2^{f}\left(1+p_{1}^{c_{1}}\right) \cdots\left(1+p_{\ell}^{c_{\ell}}\right)$ by Theorem 3.2. Note that for any $(\alpha, \beta) \in$ $\mathrm{RET}_{m, n}$ and for any integer $k, \alpha(k)=r k, \beta(2 k)=2 k, \beta(2 k+1)=2 k+1+2 s$ for some integers $r \in[m]$ and $s \in[n]$ satisfying $r^{2} \equiv 1(\bmod m), 2 s \equiv 0\left(\bmod 2^{b} q_{\ell+1}^{b_{\ell+1}} \cdots q_{\ell+g}^{b_{\ell+g}}\right)$ and for any $j=1,2, \ldots, \ell, s \equiv 0\left(\bmod p_{j}^{b_{j}}\right)$ if $r \equiv 1\left(\bmod p_{j}^{a_{j}}\right) ; s \equiv z \cdot p_{j}^{b_{j}-c_{j}}$ $\left(\bmod p_{j}^{b_{j}}\right)$ for some integer $z$ with $0 \leq z \leq p_{j}^{c_{j}}-1$ if $r \equiv-1\left(\bmod p_{j}^{a_{j}}\right)$. Let us denote such $\alpha$ and $\beta$ by $\alpha_{r}$ and $\beta_{s}$. Considering commuting rule

$$
y_{\beta}^{i} x_{\alpha}^{j}=x_{\alpha}^{\beta^{i}(j)} y_{\beta}^{\alpha^{j}(i)},
$$

one can check that the centralizer of $\left\langle x_{\alpha_{r}}, y_{\beta_{s}}\right\rangle$ is

$$
\left\{x_{\alpha_{r}}^{2 i} y_{\beta_{s}}^{j}: i \in\left[\frac{n}{2}\right], j(r-1) \equiv 0 \quad(\bmod m)\right\}=\left\langle x_{\alpha_{r}}^{2}, y_{\beta_{s}}^{k}\right\rangle
$$

where $k$ is the smallest positive integer $j$ satisfying $j(r-1) \equiv 0(\bmod m)$. This implies that for any $\left(\alpha_{r_{1}}, \beta_{s_{1}}\right),\left(\alpha_{r_{2}}, \beta_{s_{2}}\right) \in \mathrm{RET}_{m, n}$, if two groups $\left\langle x_{\alpha_{r_{1}}}, y_{\beta_{s_{1}}}\right\rangle$ and $\left\langle x_{\alpha_{r_{2}}}, y_{\beta_{s_{2}}}\right\rangle$ are isomorphic, then $r_{1}=r_{2}$. Note that

$$
\begin{aligned}
& y_{\beta_{s}} x_{\alpha_{r}}=x_{\alpha_{r}}^{\beta_{s}(1)} y_{\beta_{s}}^{\alpha_{r}(1)}=x_{\alpha_{r}}^{2 s+1} y_{\beta_{s}}^{r} \quad \text { and } \\
& y_{\beta_{s}} x_{\alpha_{r}}^{2}=x_{\alpha_{r}}^{\beta_{s}(2)} y_{\beta_{s}}^{\alpha_{r}^{2}(1)}=x_{\alpha_{r}}^{2} y_{\beta_{s}} .
\end{aligned}
$$


In fact, the above two equations determine the whole commuting rules. For any $u \in[m]$ and $v \in[n]$, if $v$ is even, then $y_{\beta_{s}}^{u} x_{\alpha_{r}}^{v}=x_{\alpha_{r}}^{v} y_{\beta_{s}}^{u}$, and if $v$ is odd, then

$$
\begin{aligned}
y_{\beta_{s}}^{u} x_{\alpha_{r}}^{v} & =x_{\alpha_{r}}^{v-1} y_{\beta_{s}}^{u} x_{\alpha_{r}}=x_{\alpha_{r}}^{v-1} y_{\beta_{s}}^{u-1} x_{\alpha_{r}}^{2 s+1} y_{\beta_{s}}^{r} \\
& =x_{\alpha_{r}}^{v-1+2 s} y_{\beta_{s}}^{u-1} x_{\alpha_{r}} y_{\beta_{s}}^{r}=x_{\alpha_{r}}^{v-1+2 s} y_{\beta_{s}}^{u-2} x_{\alpha_{r}}^{2 s+1} y_{\beta_{s}}^{2 r} \\
& =x_{\alpha_{r}}^{v-1+4 s} y_{\beta_{s}}^{u-2} x_{\alpha_{r}} y_{\beta_{s}}^{2 r}=\cdots=x_{\alpha_{r}}^{v+2 u s} y_{\beta_{s}}^{u r} .
\end{aligned}
$$

For any $v \in[n]$ with $\operatorname{gcd}(v, n)=1$,

$$
y_{\beta_{s}} x_{\alpha_{r}}^{v}=x_{\alpha_{r}}^{\beta_{s}(v)} y_{\beta_{s}}^{\alpha_{r}^{v}(1)}=x_{\alpha_{r}}^{v+2 s} y_{\beta_{s}}^{r}=x_{\alpha_{r}}^{v\left(2 v^{-1} s+1\right)} y_{\beta_{s}}^{r}
$$

because $v$ is odd, where $v^{-1}$ is an integer satisfying $v v^{-1} \equiv 1(\bmod n)$. For any $s_{1}, s_{2} \in$ $\left[\frac{n}{2}\right]$ with $\operatorname{gcd}\left(s_{1}, n\right)=\operatorname{gcd}\left(s_{2}, n\right)$, one can choose $v \in[n]$ satisfying that $\operatorname{gcd}(v, n)=1$ and $v^{-1} s_{1} \equiv s_{2}(\bmod n)$. Therefore for any $\left(\alpha_{r_{1}}, \beta_{s_{1}}\right),\left(\alpha_{r_{2}}, \beta_{s_{2}}\right) \in \mathrm{RET}_{m, n}$, if $r_{1}=r_{2}$ and $\operatorname{gcd}\left(s_{1}, n\right)=\operatorname{gcd}\left(s_{2}, n\right)$ then $\left\langle x_{\alpha_{r_{1}}}, y_{\beta_{s_{1}}}\right\rangle$ is isomorphic to $\left\langle x_{\alpha_{r_{2}}}, y_{\beta_{s_{2}}}\right\rangle$. This means that the number of non-isomorphic reflexible product of two cyclic groups of order $m$ and $n$ is at most $2^{f}\left(2+c_{1}\right) \cdots\left(2+c_{\ell}\right)$. So any reflexible product of two cyclic groups of order $m$ and $n$ is isomorphic to

$$
\left\langle x, y \mid x^{n}=y^{m}=1, y x=x^{2 s+1} y^{r}, y x^{2}=x^{2} y\right\rangle
$$

for some $r \in[m]$ and $s \in[n]$ satisfying $r^{2} \equiv 1(\bmod m), 2 s \equiv 0\left(\bmod 2^{b} q_{\ell+1}^{b_{\ell+1}} \cdots q_{\ell+g}^{b_{\ell+g}}\right)$ and for any $j=1,2, \ldots, \ell, s \equiv 0\left(\bmod p_{j}^{b_{j}}\right)$ if $r \equiv 1\left(\bmod p_{j}^{a_{j}}\right) ; s \equiv p_{j}^{b_{j}-c_{j}+z}$ $\left(\bmod p_{j}^{b_{j}}\right)$ for some integer $z=0,1, \ldots, c_{j}$ if $r \equiv-1\left(\bmod p_{j}^{a_{j}}\right)$.

Conversely, assume that for some $\left(\alpha_{r_{1}}, \beta_{s_{1}}\right),\left(\alpha_{r_{2}}, \beta_{s_{2}}\right) \in \mathrm{RET}_{m, n},\left\langle x_{\alpha_{r_{1}}}, y_{\beta_{s_{1}}}\right\rangle$ is isomorphic to $\left\langle x_{\alpha_{r_{2}}}, y_{\beta_{s_{2}}}\right\rangle$. Let $\psi:\left\langle x_{\alpha_{r_{1}}}, y_{\beta_{s_{1}}}\right\rangle \rightarrow\left\langle x_{\alpha_{r_{2}}}, y_{\beta_{s_{2}}}\right\rangle$ be an isomorphism such that $\psi\left(x_{\alpha_{r_{1}}}^{u}\right)=x_{\alpha_{r_{2}}}$ and $\psi\left(y_{\beta_{s_{1}}}^{v}\right)=y_{\beta_{s_{2}}}$.

For the remaining case, let

$$
\begin{aligned}
m & =2^{a} p_{1}^{a_{1}} p_{2}^{a_{2}} \cdots p_{\ell}^{a_{\ell}} p_{\ell+1}^{a_{\ell+1}} \cdots p_{\ell+f}^{a_{\ell+f}} \quad \text { and } \\
n & =2^{b} p_{1}^{b_{1}} p_{2}^{b_{2}} \cdots p_{\ell}^{b_{\ell}} q_{\ell+1}^{a_{\ell+1}} \cdots q_{\ell+g}^{b_{\ell+g}} \quad \text { (prime decompositions) }
\end{aligned}
$$

with $\operatorname{gcd}(m, n)=2^{c} p_{1}^{c_{1}} p_{2}^{c_{2}} \cdots p_{\ell}^{c_{\ell}}$, where $1 \leq a \leq b$ and $c_{i} \geq 1$ for any $i=1, \ldots, \ell$. For any $(\alpha, \beta) \in \operatorname{RET}_{m, n}$ and for any integer $k$,

$$
\begin{aligned}
\alpha(2 k) & =2 k t_{1}, \\
\alpha(2 k+1) & =2 k t_{1}+2 s_{1}+1, \\
\beta(2 k) & =2 k t_{2} \quad \text { and } \\
\beta(2 k+1) & =2 k t_{2}+2 s_{2}+1
\end{aligned}
$$

for some $\left(s_{1}, t_{1} ; s_{2}, t_{2}\right) \in \mathcal{Q}(m, n)$. Let $\alpha$ and $\beta$ be such permutations. Note that

$$
\begin{aligned}
& y_{\beta} x_{\alpha}=x_{\alpha}^{\beta(1)} y_{\beta}^{\alpha(1)}=x_{\alpha}^{2 s_{2}+1} y_{\beta}^{2 s_{1}+1}, \\
& y_{\beta} x_{\alpha}^{2}=x_{\alpha}^{\beta(2)} y_{\beta}^{\alpha^{2}(1)}=x_{\alpha}^{2 t_{2}} y_{\beta}^{2 s_{1}\left(t_{1}+1\right)+1}, \\
& y_{\beta}^{2} x_{\alpha}=x_{\alpha}^{\beta^{2}(1)} y_{\beta}^{\alpha(2)}=x_{\alpha}^{2 s_{2}\left(t_{2}+1\right)+1} y_{\beta}^{2 t_{1}} \text { and } \\
& y_{\beta}^{2} x_{\alpha}^{2}=x_{\alpha}^{\beta^{2}(2)} y_{\beta}^{\alpha^{2}(2)}=x_{\alpha}^{2} y_{\beta}^{2} .
\end{aligned}
$$


In fact, the above four equations determine the whole commuting rules as follows. For any $i \in[m]$ and $j \in[n]$,

$$
\begin{aligned}
y_{\beta}^{2 i} x_{\alpha}^{2 j} & =x_{\alpha}^{2 j} y_{\beta}^{2 i} \\
y_{\beta}^{2 i} x_{\alpha}^{2 j+1} & =x_{\alpha}^{2 j} y_{\beta}^{2 i} x_{\alpha}=x_{\alpha}^{2 j} y_{\beta}^{2(i-1)} x_{\alpha}^{2 s_{2}\left(t_{2}+1\right)+1} y_{\beta}^{2 t_{1}} \\
& =x_{\alpha}^{2 j+2 s_{2}\left(t_{2}+1\right)} y_{\beta}^{2(i-1)} x_{\alpha} y_{\beta}^{2 t_{1}}=\cdots=x_{\alpha}^{2 j+2 i s_{2}\left(t_{2}+1\right)+1} y_{\beta}^{2 i t_{1}} \\
y_{\beta}^{2 i+1} x_{\alpha}^{2 j} & =y_{\beta} x_{\alpha}^{2 j} y_{\beta}^{2 i}=x_{\alpha}^{2 t_{2}} y_{\beta}^{2 s_{1}\left(t_{1}+1\right)+1} x_{\alpha}^{2(j-1)} y_{\beta}^{2 i} \\
& =x_{\alpha}^{2 t_{2}} y_{\beta} x_{\alpha}^{2(j-1)} y_{\beta}^{2 i+2 s_{1}\left(t_{1}+1\right)}=\cdots=x_{\alpha}^{2 j t_{2}} y_{\beta}^{2 i+2 j s_{1}\left(t_{1}+1\right)+1} \\
y_{\beta}^{2 i+1} x_{\alpha}^{2 j+1} & =y_{\beta}^{2 i} y_{\beta} x_{\alpha} x_{\alpha}^{2 j}=y_{\beta}^{2 i} x_{\alpha}^{2 s_{2}+1} y_{\beta}^{2 s_{1}+1} x_{\alpha}^{2 j}=x_{\alpha}^{2 s_{2}} y_{\beta}^{2 i} x_{\alpha} y_{\beta} x_{\alpha}^{2 j} y_{\beta}^{2 s_{1}} \\
& =x_{\alpha}^{2 s_{2}}\left(x_{\alpha}^{2 i s_{2}\left(t_{2}+1\right)+1} y_{\beta}^{2 i t_{1}}\right)\left(x_{\alpha}^{2 j t_{2}} y_{\beta}^{2 j s_{1}\left(t_{1}+1\right)+1}\right) y_{\beta}^{2 s_{1}} \\
& =x_{\alpha}^{2 j t_{2}+2 i s_{2}\left(t_{2}+1\right)+2 s_{2}+1} y_{\beta}^{2 i t_{1}+2 j s_{1}\left(t_{1}+1\right)+2 s_{1}+1} .
\end{aligned}
$$

So any reflexible product of two cyclic groups of order $m$ and $n$ is isomorphic to

$$
\begin{aligned}
\langle x, y| x^{n}=y^{m}=1, y x=x^{2 s_{2}+1} y^{2 s_{1}+1}, y x^{2} & =x^{2 t_{2}} y^{2 s_{1}\left(t_{1}+1\right)+1}, \\
y^{2} x & \left.=x^{2 s_{2}\left(t_{2}+1\right)+1} y^{2 t_{1}}, y^{2} x^{2}=x^{2} y^{2}\right\rangle
\end{aligned}
$$

for some $\left(s_{1}, t_{1} ; s_{2}, t_{2}\right) \in \mathcal{Q}(m, n)$. In summary, we have the following theorem.

Theorem 5.1. For any positive integers $m$ and $n$, let $\Gamma$ be a group such that $\Gamma=X Y$ for some cyclic groups $X=\langle x\rangle$ of order $n$ and $Y=\langle y\rangle$ of order $m$ with $X \cap Y=\left\{1_{\Gamma}\right\}$ and there exists an automorphism of $\Gamma$ which sends $x$ and $y$ to $x^{-1}$ and $y^{-1}$, respectively.

(1) If both $m$ and $n$ are odd, $\Gamma$ is isomorphic to the abelian group $\mathbb{Z}_{m} \times \mathbb{Z}_{n}$.

(2) Let

$$
m=p_{1}^{a_{1}} \cdots p_{\ell}^{a_{\ell}} p_{\ell+1}^{a_{\ell+1}} \cdots p_{\ell+f}^{a_{\ell+f}} \quad \text { (prime factorization) }
$$

be odd and let

$$
n=2^{b} p_{1}^{b_{1}} \cdots p_{\ell}^{b_{\ell}} q_{\ell+1}^{b_{\ell+1}} \cdots q_{\ell+g}^{b_{\ell+g}} \quad \text { (prime factorization) }
$$

be even with $\operatorname{gcd}(m, n)=p_{1}^{c_{1}} \cdots p_{\ell}^{c_{\ell}}$, where $c_{i} \geq 1$ for any $i=1, \ldots, \ell$. Then $\Gamma$ is isomorphic to

$$
\left\langle x, y \mid x^{n}=y^{m}=1, y x=x^{2 s+1} y^{r}, y x^{2}=x^{2} y\right\rangle
$$

for some $r \in[m]$ and $s \in\left[\frac{n}{2}\right]$ satisfying

$$
r^{2} \equiv 1 \quad(\bmod m), \quad 2 s \equiv 0 \quad\left(\bmod 2^{b} q_{\ell+1}^{b_{\ell+1}} \cdots q_{\ell+g}^{b_{\ell+g}}\right),
$$

and for any $j=1,2, \ldots, \ell, s \equiv 0\left(\bmod p_{j}^{b_{j}}\right)$ if

$$
r \equiv 1 \quad\left(\bmod p_{j}^{a_{j}}\right), \quad s \equiv p_{j}^{b_{j}-c_{j}+z} \quad\left(\bmod p_{j}^{b_{j}}\right)
$$

for some $z=0,1, \ldots, c_{j}$ if $r \equiv-1\left(\bmod p_{j}^{a_{j}}\right)$. 
(3) Let

$$
\begin{aligned}
m & =2^{a} p_{1}^{a_{1}} \cdots p_{\ell}^{a_{\ell}} p_{\ell+1}^{a_{\ell+1}} \cdots p_{\ell+f}^{a_{\ell+f}} \quad \text { and } \\
n & =2^{b} p_{1}^{b_{1}} \cdots p_{\ell}^{b_{\ell}} q_{\ell+1}^{a_{\ell+1}} \cdots q_{\ell+g}^{b_{\ell+g}} \quad \text { (prime factorization) }
\end{aligned}
$$

with $\operatorname{gcd}(m, n)=2^{c} p_{1}^{c_{1}} p_{2}^{c_{2}} \cdots p_{\ell}^{c_{\ell}}$, where $1 \leq a \leq b$ and $c_{i} \geq 1$ for any $i=1, \ldots, \ell$. Now $\Gamma$ is isomorphic to

$$
\begin{array}{r}
\langle x, y| x^{n}=y^{m}=1, y x=x^{2 s_{2}+1} y^{2 s_{1}+1}, y x^{2}=x^{2 t_{2}} y^{2 s_{1}\left(t_{1}+1\right)+1}, \\
\left.y^{2} x=x^{2 s_{2}\left(t_{2}+1\right)+1} y^{2 t_{1}}, y^{2} x^{2}=x^{2} y^{2}\right\rangle
\end{array}
$$

for some $\left(s_{1}, t_{1} ; s_{2}, t_{2}\right) \in \mathcal{Q}(m, n)$.

For any positive integers $m$ and $n$ and for any $(\alpha, \beta),\left(\alpha^{\prime}, \beta^{\prime}\right) \in \mathrm{RET}_{m, n}$, we do not know a necessary and sufficient condition for $\left\langle x_{\alpha}, y_{\beta}\right\rangle \simeq\left\langle x_{\alpha^{\prime}}, y_{\beta^{\prime}}\right\rangle$. So we propose the following problem.

Problem 5.2. For any positive integers $m$ and $n$ and for any $(\alpha, \beta),\left(\alpha^{\prime}, \beta^{\prime}\right) \in \mathrm{RET}_{m, n}$, find a necessary and sufficient condition for $\left\langle x_{\alpha}, y_{\beta}\right\rangle \simeq\left\langle x_{\alpha^{\prime}}, y_{\beta^{\prime}}\right\rangle$. Consequently calculate the number of reflexible products of two cyclic groups of order $m$ and $n$ up to isomorphism.

\section{References}

[1] S.-F. Du, G. Jones, J. H. Kwak, R. Nedela and M. Škoviera, Regular embeddings of $K_{n, n}$ where $n$ is a power of 2. I: Metacyclic case, European J. Combin. 28 (2007), 1595-1609, doi:10.1016/j.ejc.2006.08.012.

[2] S.-F. Du, G. Jones, J. H. Kwak, R. Nedela and M. Škoviera, Regular embeddings of $K_{n, n}$ where $n$ is a power of 2. II: The non-metacyclic case, European J. Combin. 31 (2010), 1946-1956, doi:10.1016/j.ejc.2010.01.009.

[3] J. E. Graver and M. E. Watkins, Locally finite, planar, edge-transitive graphs, Mem. Amer. Math. Soc. 126 (1997), no. 601, doi:10.1090/memo/0601.

[4] G. Jones, R. Nedela and M. Škoviera, Complete bipartite graphs with a unique regular embedding, J. Comb. Theory Ser. B 98 (2008), 241-248, doi:10.1016/j.jctb.2006.07.004.

[5] G. A. Jones, Regular embeddings of complete bipartite graphs: classification and enumeration, Proc. Lond. Math. Soc. 101 (2010), 427-453, doi:10.1112/plms/pdp061.

[6] G. A. Jones, R. Nedela and M. Škoviera, Regular embeddings of $K_{n, n}$ where $n$ is an odd prime power, European J. Combin. 28 (2007), 1863-1875, doi:10.1016/j.ejc.2005.07.021.

[7] J. H. Kwak and Y. S. Kwon, Regular orientable embeddings of complete bipartite graphs, J. Graph Theory 50 (2005), 105-122, doi:10.1002/jgt.20097.

[8] J. H. Kwak and Y. S. Kwon, Classification of nonorientable regular embeddings of complete bipartite graphs, J. Comb. Theory Ser. B 101 (2011), 191-205, doi:10.1016/j.jctb.2011.03.003.

[9] Y. S. Kwon, Classification of reflexible edge-transitive embeddings of $K_{m, n}$ for odd $m, n$, East Asian Math. J. 25 (2009), 533-541, https://ynmath.jams.or.kr/jams/ download/KCI_FI001404071.pdf.

[10] R. Nedela, M. Škoviera and A. Zlatoš, Regular embeddings of complete bipartite graphs, Discrete Math. 258 (2002), 379-381, doi:10.1016/s0012-365x(02)00539-3. 